\title{
Sounding Rocket Roll Control Through DBD Plasma Actuator Boundary Layer Control
}

\author{
by \\ Spencer Ryan Deehr Sumanik \\ A Thesis submitted to \\ the Faculty of Graduate Studies and Research \\ in partial fulfilment of \\ the requirements for the degree of \\ Master of Applied Science \\ in \\ Aerospace Engineering \\ Ottawa-Carleton Institute for Mechanical and Aerospace Engineering \\ Carleton University \\ Ottawa, Ontario, Canada \\ January 19th 2018 \\ Copyright (0) \\ 2018 - Spencer Ryan Deehr Sumanik
}




\section{Abstract}

Dielectric Barrier Discharge (DBD) plasma actuators induce a plasma flow that alters the velocity of the surrounding air across a surface. The induced flow, directed tangential to the surface, inputs momentum to the boundary layer. This analysis examines the feasibility of a simplified boundary condition within computational fluid dynamic (CFD) simulations of a DBD plasma actuator mounted on a rocket fin. The simulations examine the location of the plasma actuator through a range of freestream velocities. Resulting in a plasma actuator location between $40 \%$ and $45 \%$ of the fin chord producing the most force. With the actuator at $50 \%$ chord a velocity range between 10 and $90 \mathrm{~m} / \mathrm{s}$ is tested. The highest normal force experienced by the fin due to plasma actuation is approximately $5.5 \mathrm{mN} / \mathrm{m}$ at a freestream $70 \mathrm{~m} / \mathrm{s}$ decreasing as the rocket decelerates. This force and the resulting torque induces an angular velocity of 2.86 RPM of roll on a sounding rocket. 


\section{Acknowledgments}

This master's degree may not have been possible without the help of many people supporting me in many different ways. Specifically, I would like to thank my supervisor, Dr. Jason Etele, for his guidance and support. A special thank you to Matt Mills for his help with the first simulations that built the foundations of the results

presented here. I also send my thanks to Dr. Bruce Colpits of University of New Brunswick for his unending supply of answers to the barrage of questions I sent his way. By no means is this list complete, and I hope that those not mentioned here know I appreciate their support, thanks for the coffee. 


\section{Table of Contents}

Abstract $\quad$ ii

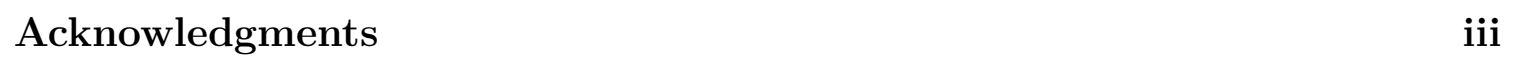

List of Tables $\quad$ vii

List of Figures $\quad$ viii

List of Acronyms $\quad$ x

List of Symbols $\quad$ xi

1 Introduction 1

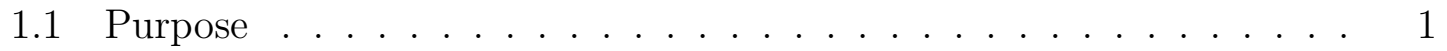

1.2 Plasma Actuators . . . . . . . . . . . . . . . . . 3

1.2.1 Corona Discharge Plasma Actuators . . . . . . . . . . . . 3

1.2.2 Dielectric Barrier Discharge Plasma Actuators . . . . . . . . . 4

1.2.3 Single Dielectric Barrier Discharge Plasma Actuators . . . . . 6

1.2.4 Orientation and Placement of DBD Plasma Actuator . . . . . 7

1.3 Actuator Configuration and Electrical Input . . . . . . . . . . . . 15 
1.4 Aerodynamic Placement of the Actuator . . . . . . . . . . . . . . . 20

1.5 Actuator Operational Freestream Velocity Range . . . . . . . . . . . 22

1.6 Roll Control Using Plasma Actuators . . . . . . . . . . . . . . . . . . 24

1.7 Relevance ........................ . . 26

2 Computational Theory 28

2.1 OpenFOAM . . . . . . . . . . . . . . . . . 28

2.2 Navier-Stokes Equations . . . . . . . . . . . . . . . . . . . . . 29

2.3 Turbulence Modelling . . . . . . . . . . . . . . . . . . . . 43

3 Grid Refinement Study $\quad 46$

3.1 Two Dimensional Computational Domain . . . . . . . . . . . . 46

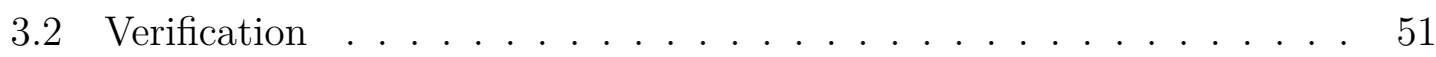

3.2.1 Blasius Comparison . . . . . . . . . . . . . 51

3.2.2 Turbulent Boundary Layer Comparison . . . . . . . . . . . . . 58

3.3 Validation ....................... 62

3.4 Mesh Convergence .................... . . 65

$\begin{array}{lll}4 & \text { Results } & 69\end{array}$

4.1 Actuator Location . . . . . . . . . . . . . . . . . 70

4.2 Normal Force with Respect to Velocity . . . . . . . . . . . . . . . . . 75

4.3 Predicting Induced Angular Velocity . . . . . . . . . . . . . . . . 79

5 Conclusions and Recommendations $\quad 89$

5.1 Conclusions . . . . . . . . . . . . . . . . . . 89

5.2 Recommendations . . . . . . . . . . . . . . . . . . . . 91 
List of References 


\section{List of Tables}

1 Boundary conditions for computational domain . . . . . . . . 50

2 Course, medium and fine mesh details . . . . . . . . . . . . 65 


\section{List of Figures}

1 Basic DBD plasma actuator schematic $\ldots \ldots \ldots \ldots \ldots$

2 General description of OpenFOAM layout [44] . . . . . . . . . . 29

3 Simple finite volume grid with annotation $\ldots \ldots \ldots \ldots$

4 As-built sounding rocket fin . . . . . . . . . . . . 47

5 Computational domain of the simulation . . . . . . . . . . 50

6 Comparison to the Blasius Solution . . . . . . . . . . . 57

$7 \quad$ Van Driest turbulent boundary layer model and simulation data. . . . 61

8 Quiescent flow plasma actuator test . . . . . . . . . . . . . . 63

9 Quiescent flow plasma actuator test . . . . . . . . . . . . . . . 64

10 Grid convergence test of quiescent boundary layer . . . . . . . . 66

11 Pressure distributions at $20 \mathrm{~m} / \mathrm{s} \ldots \ldots \ldots \ldots \ldots$

12 Contour plot of pressure around plasma actuator . . . . . . . 73

13 Force per meter with respect to chord $20 \mathrm{~m} / \mathrm{s} \ldots \ldots \ldots . . \ldots 74$

14 Force per meter with respect to chord $70 \mathrm{~m} / \mathrm{s} \ldots \ldots \ldots$

15 Force with respect to velocity $\ldots \ldots \ldots \ldots \ldots \ldots$

16 Sounding rocket dimensions $\ldots \ldots \ldots \ldots$. . . . . . . 81

17 Component parts of acceleration and velocity . . . . . . . . 84

viii 
18 Rocket velocity with respect to time. . . . . . . . . . . 86

19 Altitude with respect to time . . . . . . . . . . . . . 87

20 Angular velocity with respect to time . . . . . . . . . . . . . . 88 


\section{List of Acronyms}

\begin{tabular}{ll}
\hline Acronyms & Definition \\
\hline AoA & Angle of Attack \\
CFD & Computational Fluid Dynamics \\
DBD & Dielectric Barrier Discharge \\
EHD & Electrohydrodynamic \\
IMU & Inertial Measurement Unit \\
P-P & Peak to Peak \\
PIV & Particle Image Velocimetry \\
PMMA & Polymethyl Methacrylate or Plexiglas \\
RANS & Reynolds Averaged Navier-Stokes \\
SDBD & Single Dielectric Barrier Discharge \\
SIMPLE & Semi-Implicit Method for Pressure Linked Equations \\
SST & Shear Stress Transport \\
UNB & University of New Brunswick \\
\hline
\end{tabular}




\section{List of Symbols}

\begin{tabular}{|c|c|c|}
\hline Symbols & Definition & Units \\
\hline$a$ & Acceleration & $m / s^{2}$ \\
\hline$b$ & Span & $m$ \\
\hline$C_{D}$ & Coefficient of Drag & \\
\hline$C_{L}$ & Coefficient of Lift & \\
\hline$C_{p}$ & Coefficient of Pressure & \\
\hline$C_{s d}$ & Coefficient of Skin Friction Drag & \\
\hline$E$ & Total Energy & $J$ \\
\hline$F$ & Force & $\mathrm{kgm} / \mathrm{s}^{2}$ \\
\hline$H$ & Boundary Layer Shape Factor & \\
\hline$I$ & Inertia & $\mathrm{kgm}^{2}$ \\
\hline$k$ & Turbulent Kinetic Energy & $J$ \\
\hline$L$ & Torque Arm Length & $m$ \\
\hline$m$ & Mass & $k g$ \\
\hline$P$ & Pressure & $P a$ \\
\hline$q$ & Dynamic Pressure & $P a$ \\
\hline
\end{tabular}




\begin{tabular}{|c|c|c|}
\hline$r$ & Radius & $m$ \\
\hline$r_{a}$ & Length of Moment Arm & $m$ \\
\hline$R_{e}$ & Reynolds Number & \\
\hline$S$ & Surface Area & $m^{2}$ \\
\hline$t$ & Time & $s$ \\
\hline$u$ & X Component of Velocity & $m / s$ \\
\hline$U$ & Freestream Velocity & $m / s$ \\
\hline$v$ & Y Component of Velocity & $m / s$ \\
\hline$x$ & Distance in $\mathrm{X}$ direction & $m$ \\
\hline$X$ & Horizontal Distance Value & $m$ \\
\hline$y$ & Distance in Y Direction & $m$ \\
\hline$y^{+}$ & Non-dimensional Distance from Surface to First Node & \\
\hline$Z$ & Vertical Distance Value & $m$ \\
\hline$\alpha$ & Angle of Attack & rad \\
\hline$\beta$ & Turbulence Model Constant & \\
\hline$\beta_{p}$ & Correction Factor & \\
\hline$\Gamma$ & Circulation & $m^{2} / s$ \\
\hline$\delta$ & Boundary Layer Thickness & $m$ \\
\hline$\eta$ & Blasius Self Similar Variable Equation 42 & \\
\hline$\theta$ & Flight Path Angle & rad \\
\hline$\mu$ & Dynamic Viscosity & $k g /(m \cdot s)$ \\
\hline$\nu$ & Kinematic Viscosity & $m^{2} / s$ \\
\hline$\rho$ & Density & $\mathrm{kg} / \mathrm{m}^{3}$ \\
\hline
\end{tabular}




\section{$\tau$}

$\phi$

$\omega$

Accents

Torque $\quad \mathrm{Nm}$

Roll Angle rad

Turbulent Dissipation Rate $1 / s$

$\begin{array}{ll}\cdot & \text { Rate } \\ . & \text { Change of Rate }\end{array}$

\section{Superscript}

$\begin{array}{ll}* & \text { Initial Value } \\ n & \text { Iteration Value }\end{array}$

\section{Subscript}

\begin{tabular}{ll}
$\infty$ & Freestream \\
$i$ & Node Index in X Direction \\
$j$ & Node Index in Y Direction \\
$t$ & Turbulent Eddy \\
\hline
\end{tabular}




\section{Chapter 1}

\section{Introduction}

\subsection{Purpose}

The representation of dielectric barrier discharge (DBD) plasma actuators within computational fluid dynamic (CFD) simulations is a method of examining a wide range of parameters without the expense of performing physical tests. The purpose of this thesis is to examine the feasibility of implementing an a-physical boundary condition to represent the physical system that is the DBD plasma actuator. This boundary condition is implemented as a section of a flat plate with a set surface velocity tangential to the surface, while the remainder of the plate maintains the no slip condition. This method of modelling the DBD plasma actuator is simple, requiring no changes to the structure of the solver, and can be implemented in almost any simulation.

The intended use of this boundary condition in this analysis is to explore the

feasibility of a DBD plasma actuator to induce roll a sounding rocket. Sounding 
rockets are suborbital unguided rockets that carry scientific payloads along parabolic paths [1]. Many sounding rockets are imbued with a specific amount of angular velocity to provide spin stabilization, typically through mounting the rocket fins with an angle of attack (AoA) such that a torque is applied an angular velocity is achieved. Inducing this roll through plasma actuation allows for a measure of control over the angular velocity of the rocket.

A future flight test of a sounding rocket is planned in partnership with the University of New Brunswick (UNB). The rocket will have a DBD plasma actuator mounted on each of the three fins for the purpose of inducing roll on the rocket. The proposed boundary condition is implemented in CFD simulations to estimate the resulting angular velocity of this rocket during its flight from engine burnout to apogee. This is done through two main tests. The first is the examination of the boundary condition applied through a range of chordwise locations to find the local maximum force that is applied to the fin. The second is to test the boundary condition at one chordwise location of $50 \%$ chord, based on the as-built fin provided by UNB, through a range of velocities to create a force with respect to velocity curve.

This force with respect to velocity curve is paired with the predicted trajectory of the rocket to find the final angular velocity. This analysis is performed to provide a predictive basis for the induced roll rate. While also ensuring that the inertial measurement units (IMU) on board the rocket are capable of observing the induced roll rate and have an appropriate dynamic range to effectively measure the angular velocity. 


\subsection{Plasma Actuators}

Plasma is the fourth state of matter consisting of equal parts positively charged particles and free electrons. The net charge of the plasma is therefore neutral. Plasma can be found naturally in phenomena such as the aurora, or it can be manufactured through a variety of methods. Plasma actuators are one such method of producing a plasma.

Plasma's ionic nature allows it to be manipulated by electric or magnetic fields. Electrohydrodynamic (EHD) devices are capable of producing and maintaining a stable plasma discharge in air at atmospheric pressure. The operation of the EHD device and the resulting electric field induces a velocity on the charged particles. Collisions between the charge particles and the neutral gas molecules transfer momentum to the surrounding air. The ability to directly transform electric energy to kinetic energy in surrounding air makes plasma actuators an area of interest

for aerodynamic control systems. Two main types of EHD devices exist: corona discharge plasma actuators and DBD plasma actuators.

\subsubsection{Corona Discharge Plasma Actuators}

The corona discharge type of plasma actuator uses two electrodes exposed to air mounted on a dielectric surface, and separated by a distance. A direct current 
is applied to one of the electrodes, allowing for a discharge of plasma arcing from one electrode to the other. During the plasma discharge positively charged particles accumulate around the anode while electrons are produced by the cathode. The positively charged particles are exchanged with the electrons and momentum is transferred between the charged particles and the neutral surrounding gas [2]. Due to the positively charged particles having more mass than the electrons the result is an induced flow of the air molecules in the direction of the cathode.

\subsubsection{Dielectric Barrier Discharge Plasma Actuators}

The DBD plasma actuators consist of two electrodes separated by a layer of dielectric material Figure 1. With the application of an alternating current to the electrodes the air molecules are ionized by the strong electric field produced between the exposed electrode and the encapsulated electrode. The newly ionized particles then experience a force applied by the electric field. This force accelerates ions inducing a velocity in the plasma, estimated to be on the order of $100 \mathrm{~m} / \mathrm{s}$ [3]. The ions then collide with the neutral gas transferring momentum to the gas inducing a flow. The force applied to the ions is shown to produce small levels of thrust by Enloe et al. [4]. The thrust originates from the plasma applying a body force to the surrounding gas that in turn applies a force to the electrodes through the plasma actuator's electric field.

The exposed electrode of a DBD plasma actuator will transfer electrons if its charge is greater than the charge of the dielectric material. The discharge of electrons will 


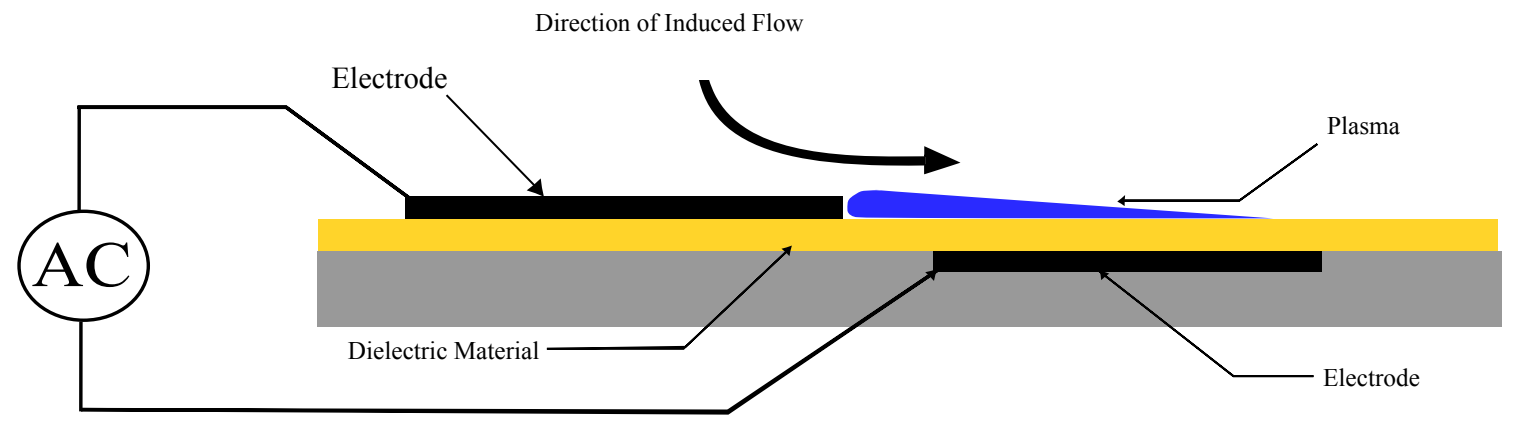

Figure 1: A basic schematic of a surface mounted DBD actuator and the induced flow through the creation of plasma and the presence of the electric field.

terminate unless this charge is continually increased. The application of alternating current to the electrode breaks the cycle into two stages. The first stage of the cycle occurs when the exposed electrode has a greater negative charge than the surface of the dielectric material. When the potential difference reaches high enough levels, the exposed electrode can emit electrons. These electrons reach an equilibrium in the charge between the exposed electrode and the dielectric unless the voltage of the electrode is continually increased. The second stage of the cycle occurs when the charge of the exposed electrode reverses, this pulls the charge off of the dielectric surface. The charge is limited to the charge deposited on the surface from the first half of the cycle [3][5].

During the $\mathrm{AC}$ cycle, the air ionizes twice, however the ionization during each half cycle is not symmetric. When the exposed electrode has negative charge the discharge of plasma is uniform, while during positive charge of the exposed electrode the plasma discharge is a series of smaller more intense discharges providing a fragmentary plasma. The asymmetry of the cycle is what allows for the plasma 
actuator to transfer momentum to the surrounding air [6].

\subsubsection{Single Dielectric Barrier Discharge Plasma Actuators}

Single dielectric barrier discharge (SDBD) plasma actuators can direct the plasma parallel to the surface to which they are mounted, as shown in Figure 1. The induced velocity can be created in still air to create an induced velocity profile or with a freestream velocity to change the velocity profile within a boundary layer. This configuration is shown in Figure 1 with the direction of the induced flow

determined through calculation of a vector field by Enloe [4], and particle image velocimetry (PIV) by Post [7]. The SDBD creating this particular flow pattern is also observed by others [6][8][9].

In still air, the plasma actuator moves air downward over the top of the actuator and accelerating it parallel to the surface. The plasma actuator alters the velocity profile near to the surface, within the magnitude of a few millimetres above the surface to which it is mounted. This allows the actuator to alter the behaviour of the fluid flow, including: reducing drag, attaching naturally separated flow, separating naturally attached flow or increasing the vorticity and circulation near the surface. 


\subsubsection{Orientation and Placement of DBD Plasma Actuator}

The root of the changes in motion induced on the surrounding fluid stems from the change in momentum near the surface. The change in fluid flow behaviour over the surface is dependent on the orientation of the actuator relative to the freestream velocity.

For example, DBD plasma actuators oriented such that the induced velocity is directed perpendicular to the freestream flow produce stream-wise vortices. The vortices are created due to the increase in velocity near the surface producing a low pressure zone and pulling the surrounding fluid from the behind and above the actuator. This results in the creation of a vortex above the plasma actuator. These vortices are shown to be of similar strength to vortices produced by vane type vortex generators sometimes placed on airfoils [10][11]. The advantage of DBD plasma actuators for use for vortex generation is that DBDs are capable of producing vortexes in quiescent air, while vanes require a pressure differential between the windward and leeward sides of the vane. The strength of these vortexes is shown to be directly influenced by the strength of the actuation [12]. However, it was concluded that at high altitude the DBD plasma actuators may be less effective due to lower atmospheric pressure.

At lower pressure the number of molecules per cubic meter is reduced. This not only increases the difficulty for plasma to be created in the fluid, but also reduces the number of collisions between the positive ions and the neutral fluid. Both these factors reduce the total momentum imparted on the gas decreasing the effectiveness 
of the actuator.

Actuators placed such that the induced velocity is perpendicular to that of the freestream flow is also shown to increase the drag coefficient and propagation of vortices near the surface that continue downstream. Furthermore, it was noted by Roth [13] that the boundary layer thickness is altered due to plasma actuation. Alterations in the boundary layer thickness were also observed by Jukes and Choi [11] and Jianyang [14][15].

Placing the plasma actuator such that the induced flow is oriented parallel to the freestream has various effects on the flow, including, but not limited to, drag reduction, boundary layer manipulation and flow attachment. Many researchers examine the advantages of plasma actuators placed in this configuration. This orientation of the plasma actuator increases the momentum within the boundary layer, decreasing the likelihood of flow separation and changing the behaviour of the flow such that the plasma actuators may be capable of replacing or increasing the efficiency of control surfaces such as flaps, slats or ailerons.

Roth and Sherman [13][16] prove that the drag on a flat plate can be reduced by orienting the plasma actuator in the direction of the freestream. By increasing the momentum close to the surface of the flat plate the shear force experienced by the plate is reduced. The actuators are placed in series with a spacing of $8.5 \mathrm{~mm}$. The drag is shown to be reduced through all flow velocities tested in both laminar and turbulent flow regimes. 
Moreau et al. [17] observe a reduction in drag on a flat plate with a DC surface corona discharge plasma actuator embedded flush with the surface at the $50 \%$ chord location. At all examined freestream velocities, between $5 \mathrm{~m} / \mathrm{s}$ and $25 \mathrm{~m} / \mathrm{s}$, the reduction in drag is held fairly constant at approximately $20 \mathrm{mN}$.

Decreases in drag on a NACA 0015 airfoil at $20 \mathrm{~m} / \mathrm{s}$ are examined by Bernard et al. [18] with a DBD plasma actuator. The plasma actuator is placed at the leading edge of the airfoil with the induced velocity directed in the same direction of as the freestream flow. The lift and drag are measured using a force balance while the flow is observed with a PIV system. The plasma actuator produces a decrease in drag coefficient of approximately $\Delta C_{d}=0.02$ from an initial value of $C_{d}=0.22$ at an AoA of $16^{\circ}$. Larger changes in drag coefficient are observed at higher angles of attack, specifically past the point of flow separation.

The plasma actuator orientation used above is capable of maintaining an attached flow past the angle of natural separation on both a flat plate, [19], and an airfoil [20]. The presence of induced velocity within the boundary layer in the direction of the freestream flow increases the momentum in the slower moving fluid close to the surface. The momentum addition can reduce adverse pressure gradients in boundary layers reattaching separated flow to a surface and increasing the AoA at which flow separation occurs [9][21].

This is shown to be true on a NACA 0015 airfoil at an AoA beyond the natural 
point of flow separation. Plasma actuators mounted to the suction side of the airfoil add momentum to the lower regions of the boundary layer reducing the adverse pressure gradients that induce flow separation. As the flow can remain attached at AoA before flow separation occurs, an increase in lift coefficient is observed [22][23].

Low Reynolds number experiments in laminar boundary layers by Sosa and Artana [24] examine flow reattachment using a corona discharge plasma actuator mounted on a NACA 0015 airfoil. Activation of the plasma actuator shows a decrease in pressure coefficient on the suction side of the airfoil. Smoke stream flow visualization shows that the flow successfully re-attaches to the surface of the airfoil.

Plasma actuators mounted up-stream of the point of natural separation on a convex separation ramp can successfully reattach the flow of a laminar boundary layer [25]. The reattachment is measured through calculation of the boundary layer shape factor. Un-actuated cases show shape factor values of $H=3.03$ indicating separation and activation of the plasma actuators decreases the shape factor to $H=1.51$ which is within the range of attached flow. Similar separation ramp attachment is produced through measuring the change in lifting force on the wing to which the ramp is mounted [26].

Greenblatt et al. examine flow over a flat plate at low Reynolds numbers [19] to examine the increase of lift for micro aerial vehicles which can experience low Reynolds number flow and often use thin flat plates for aerodynamic structures and lifting surfaces. With the actuator inducing velocity in the same direction as the 
freestream, the results show attachment of separated flow and an increase of lift coefficient on flat plates at AoA beyond the point of natural separation. Increases in lift coefficient are observed for all AoAs after the point of natural separation for all examined Reynolds numbers.

DBD plasma actuated flow separation control over a RAE 5212 swept wing with a sweep angle of $25^{\circ}$ is examined for flow separation [27]. In the un-actuated cases separation originates at the fin tip at an AoA of approximately $16^{\circ}$ and propagates toward the root as the AoA is increased to $19^{\circ}$. With the activation of a plasma actuator mounted $9 \mathrm{~mm}$ from the leading edge, the flow is shown to resist separation up to an AoA of $19^{\circ}$, after which the flow separates quickly across the whole wing.

Unlike a typical airfoil or wing, delta wings use three dimensional vortices to produce lift. Delta wings are still subject to stalls when the primary vortex breaks down, culminating in a drastic and abrupt reduction of lift production by the wings. DBD plasma actuators can be used to reduce the unfavourable pressure gradients that can lead to vortex breakdown. Plasma actuators inducing flow in the same direction as the direction of the freestream increase the coefficient of lift by preventing vortex breakdown [28]. The leading edge plasma actuator configuration is shown to produce a jet direction away from the leading edge towards the center of the wing. Flow visualizations using PIV methods show that at an AoA of $36^{\circ}$ there is no vortex over the wing in the control case. Activating the plasma actuator rekindles the vortex over the wing increasing the lift coefficient. Throughout all tested AoA an increase in lift coefficient is observed. However, the increase is substantially more 
significant at higher angles of attack that occur within the post stall environment of the wing $\left(A o A \approx 26^{\circ}\right)$.

Placing the plasma actuator such that the induced velocity is in the opposite direction of the freestream flow also has advantageous aerodynamic applications. This orientation of the plasma actuator can prevent vortex breakdown on delta wings by adding momentum to the vortex structures on the surface. Plasma actuators can also prematurely separate flow from a surface by introducing an adverse pressure gradient to the aerodynamic surface.

On a delta wing, placing the plasma actuators such that the flow is induced perpendicular to the leading edge, inducing flow upstream, proves to breakdown the vortex farther upstream than the natural breakdown location. Thus, reducing the lift coefficient. This is likely due to the plasma actuator countering the flow over the wing and perturbing the attached vortices. With the plasma actuators oriented parallel to the leading edge, inducing flow upstream and toward the leading edge, stabilized vortices are produced downstream of the natural breakdown point [29]. The plasma actuators introduce momentum near the line of secondary separation introducing a pressure gradient that encourages separation, this promotes vortex stabilization and prevents vortex breakdown.

Bernard [30] examines the separation of internal flow in a diverging circular pipe. The angle of divergence of the nozzle is set to $12^{\circ}$. The plasma actuator is placed near the leading edge of the diverging surface. Three dimensional flow structures 
are examined with the plasma actuator off and compared to those of the flow with the plasma actuator on. An experimental PIV system is used to measure the flow velocity in three dimensions. The resulting flow detachment caused by the plasma actuator alters the velocity profile beyond the outlet of the diffuser. The detachment changes the flow behaviour such that the flow velocity is reduced near the surface when compared to the control observable at all tested velocities. The x-component of velocity (the direction of the flow in the pipe) shows a portion of zero velocity near the surface of the diffuser indicating that there is a region of separated flow.

The experiment also shows that inducing flow in the opposite direction of the freestream increases the number of vortices within the flow. The origin of these vortices is likely due to greater oscillation within the shear layer as a result of the plasma actuator. A clockwise rotation is observed in the large scale vortices resulting in negative circulation. Two prominent vortices appear to propagate in the flow, one immediately after the plasma actuator, and another downstream of the outlet. Averaging the total number of vortices present over the experiment, twice the number of vortices are observed at $10 \mathrm{~m} / \mathrm{s}$ with the DBD plasma actuator active as compared to the control. This increase in vortices decreases with increasing velocity.

A similar experiment is performed by Labergue et al. [2] examining the use of a surface corona discharge plasma actuator to separate flow in a diffuser at $5 \mathrm{~m} / \mathrm{s}$ and an angle of $27^{\circ}$. It is shown that the corona discharge plasma actuator can successfully detach the flow from the surface. This experiment builds on Labergue's 
earlier work in 2004 [31] where it is shown that flow can be detached from a flat plate at an AoA of $17^{\circ}$.

Later work by Labergue et al. [32] compares surface corona discharge actuators to DBD actuators in separating the flow from a diverging nozzle. The diffuser used in the experiment can be adjusted on both the upper and lower surfaces. The DBD actuator is operated such that the induced velocity directed upstream. The experiment uses PIV to observe the flow field in the diffuser. At jet velocities of 10 $\mathrm{m} / \mathrm{s}$ the DBD plasma actuator is found to be more effective at separating the flow from the surface, while also having more homogeneous plasma distribution across the exposed electrode when compared to corona discharge plasma actuators.

Labergue [32] shows that at a freestream velocity of $10 \mathrm{~m} / \mathrm{s}$ the flow remains attached to the diffuser at an angle of $16^{\circ}$ for the non-actuated case. Activation of the plasma actuator induces flow separation from the diffuser wall. The experiment is repeated through a range of velocities at an angle of $17^{\circ}$. Flow separation is induced up to a velocity of $15 \mathrm{~m} / \mathrm{s}$. However, the flow only experiences a minor decrease in velocity near the surface at freestream velocities of $20 \mathrm{~m} / \mathrm{s}$ and $30 \mathrm{~m} / \mathrm{s}$. Furthermore, it is found that farther away from the center line of the actuator, the flow is more attached indicating that the plasma actuator can separate the flow behind it, but has little effect in the span-wise direction.

Flight tests with a plasma actuator placed on the leading edge in a counter flow arrangement on an un-powered aircraft SZD 48-3 Jantar Std. are performed by 
Sidorenko et al. [33]. Tufts placed on the wing show the flow direction, and to examine the dynamics of the flow separation test stalls were performed in flight. Videos of the test stalls show that the separation bubble moves from the trailing edge to the leading edge as the AoA is increased. The instrument panel places the aircraft at an airspeed of $73 \mathrm{~km} / \mathrm{h}$ or approximately $20 \mathrm{~m} / \mathrm{s}$, this is a representative measurement of the freestream flow velocity. Upon switching on the actuator a separation bubble can be observed at the trailing edge of the wing. Full separation of the flow from the wing was not observed.

\subsection{Actuator Configuration and Electrical Input}

Apart from the orientation of the induced velocity, the plasma actuator also shows different behaviours depending on: the nature of the electrical input (voltage and frequency), as well as the dielectric thickness, electrode separation and electrode dimensions. Experiments are performed pertaining to the nature of the plasma actuator varying physical parameters such as electrode gap, electrode width, and dielectric material thickness. All of which have proven to have an effect on the induced velocity.

Van Dyken et al. [34] measures the force produced through plasma actuation with a mass balance scale. It is found that the shape of the waveform input into the plasma actuator effects the amount of thrust produced. As a function of power, a positive saw tooth wave function produces the most thrust followed by the sine wave 
form, while the square wave produces the least amount of thrust. As mentioned earlier, the flow of ions is only present if the charge of the exposed electrode is continually changed. The square wave having sections of zero gradient of charge will produce less induced thrust during these sections of the waveform. The sine wave has small sections of zero gradient to allow for near continuous change in charge, while the saw tooth waveform has a continual change in charge throughout.

Further experimentation by Van Dyken et al. [34] examines the thickness of the dielectric material and the resulting thrust produced by the actuator, using the dielectric material Kapton. It is shown that thinner dielectric layers produced higher thrust values. However, these thinner materials would breakdown as the voltage was increased. At a P-P voltage of $8 \mathrm{kV}$ the $8 \mathrm{~mm}$ configuration produces $5 \times 10^{-4} \mathrm{~kg}$ of force compared to the $4 \times 10^{-4} \mathrm{~kg}$ of the $12 \mathrm{~mm}$ counterpart. However, at higher voltage the $12 \mathrm{~mm}$ dielectric is capable of producing $6 \times 10^{-4} \mathrm{~kg}$ of force at $10 \mathrm{kV}$, a high enough voltage to break down the thinner dielectric. An upper limit to the dielectric thickness is predicted, but it was not observed in these experiments.

Experimentation by Enloe et al. [3] states that the plasma expands forward from the exposed electrode on the order of $100 \mathrm{~m} / \mathrm{s}$. The velocity of the expansion is not strongly dependent on the applied voltage. However, the distance downstream that the plasma extends is dependent on this voltage. It is observed that a voltage range between $5 \mathrm{kV}$ and $9 \mathrm{kV}$ varies the length of the plasma discharge downstream of the exposed electrode from $3 \mathrm{~mm}$ to $8 \mathrm{~mm}$ respectively. 
Two independent research teams of Forte et al. [35] and Roth \& Dai [36] perform extensive studies into plasma actuator structures. Both parties explore a range of parameters with regards to the electrode gap, electrode thickness, frequency and voltage.

Varying the distance of the electrode gap Forte et al. [35] found that for voltage of $20 \mathrm{kV}$, a frequency of $700 \mathrm{~Hz}$ and a dielectric material of Polymethyl Methacrylate (PMMA), the highest induced velocity of $4 \mathrm{~m} / \mathrm{s}$ is produced with a gap of $5 \mathrm{~mm}$. Decreasing this distance creates a symmetry of the electric field decreasing the ability to accelerate ions. While increasing the distance between electrodes decreases the induced velocity due to the movement of the electric field into the dielectric.

Exploring the dimensions of the electrodes, Forte et al. finds that increasing the width of the encapsulated electrode creates an asymptotic curve, with a width of 20 mm being the optimal dimension [35]. The application of $20 \mathrm{kV}$ and $700 \mathrm{~Hz}$ applied to the actuator a velocity of approximately $5 \mathrm{~m} / \mathrm{s}$ is produced. Increasing the electrode width beyond $20 \mathrm{~mm}$ is ineffective as the voltage across the plasma is no longer high enough to sustain the discharge. Similar asymptotic results are found by other researchers with a voltage of $16 \mathrm{kV}$ peak to peak $(\mathrm{P}-\mathrm{P})$ input to the actuator [5].

Forte et al. [35] also explores the effect of frequency and voltage on the induced velocity of the plasma actuator. Varying the input frequency between $100 \mathrm{~Hz}$ and $1200 \mathrm{~Hz}$ with voltages between $8 \mathrm{kV}$ to $25 \mathrm{kV}$. PMMA is used for the dielectric material and an electrode width of $20 \mathrm{~mm}$ and no electrode gap. Forte et al. finds 
that with a frequency of $1 \mathrm{kHz}$ and increasing the voltage increases the induced velocity due to higher Coulomb forces. Furthermore, holding a constant voltage of $20 \mathrm{kV}$ and increasing the frequency also increases the induced velocity along an asymptotic curve reaching velocities of $6.5 \mathrm{~m} / \mathrm{s}$. This increase in velocity is likely due to steeper gradients in the applied waveform as well as a greater number of collisions of ions with the neutral gas.

Roth and Dai [36] perform similar experiments examining the highest induced velocities at frequencies between $5 \mathrm{kHz}$ and $10 \mathrm{kHz}$ and voltages of $4 \mathrm{kV}$ to 10 $\mathrm{kV}$ with a dielectric material of Teflon. Roth and Dai experiment with electrode separation distance and varying the electrode gap between $0 \mathrm{~mm}$ and $3 \mathrm{~mm}$ through the full range of frequencies and two voltages of $7 \mathrm{kV}$ and $9 \mathrm{kV}$. An electrode gap of $2 \mathrm{~mm}$ produces the highest induced velocities through all tested frequencies and voltages, with a maximum velocity of $5.5 \mathrm{~m} / \mathrm{s}$ at $8 \mathrm{kHz}$ and $9 \mathrm{kV}$. As the gap increases from zero, the induced velocity increases before reaching a maximum and beginning to reduce. The increase and decreasing trend of induced velocity with respect to electrode gap is also observed by Pons et al. [37].

Roth and Dai also examine the effects of varying frequency and voltage on the maximum induced velocity. The experiment uses a Teflon dielectric and no electrode gap. The experiment examines each frequency, $5 \mathrm{kHz}$ to $10 \mathrm{kHz}$ in steps of $1 \mathrm{kHz}$, through a range of voltages, $4 \mathrm{kV}$ to $10 \mathrm{kV}$ in steps of $1 \mathrm{kV}$. The experiment shows that an optimum frequency is present for each examined voltage. For the range of parameters explored by Roth and Dai, a maximum velocity of $5 \mathrm{~m} / \mathrm{s}$ is found with 
a frequency of $5 \mathrm{kHz}$ and at $8 \mathrm{kV}[36]$.

Maslov et al. [27] examine the operating frequency of the actuator. At an AoA of $18^{\circ}$ it was found that ranging the frequency from $50 \mathrm{~Hz}$ to $3000 \mathrm{~Hz}$ and holding the voltage steady at $10 \mathrm{kV}$ P-P, the coefficient of pressure, $C_{p}$, over the airfoil can be altered. The coefficient of pressure sees little change between $50 \mathrm{~Hz}$ and 800 $\mathrm{Hz}$, from approximately $C_{p}=-1$ to $C_{p}=-1.5$ respectively. The small decrease indicates that the flow is not completely reattached to the wing. Testing at $1 \mathrm{kHz}$ shows a decrease of the coefficient of pressure to approximately $C_{p}=-3.5$ indicating a completely reattached flow to the surface. The value of $C_{p}$ continues to decrease with increasing frequency, up to $2 \mathrm{kHz}$ before increasing back to $C_{p}=-1.5$ at a frequency of $2.2 \mathrm{kHz}$, and remains there through to the maximum tested frequency of $3 \mathrm{kHz}$.

Research by Enloe et al. [5] explores the dimensions of the exposed electrode, concluding that smaller exposed electrodes produce greater thrust. More specifically a wire electrode of radius $0.19 \mathrm{~mm}$ produces nearly twice as much thrust per watt as an electrode of radius $0.5 \mathrm{~mm}$. Similar trends are observed with exposed electrodes made of foil. This is thought to originate from the enhancement of the electrical field near the surface of the exposed electrode being greater with reduced radius. 


\subsection{Aerodynamic Placement of the Actuator}

The location of the plasma actuator on the surface of the aerodynamic body has an effect on the behaviour of the flow. Placement of the actuator on the leading edge of a surface, the mid-chord, and trailing edge of the body alters the flow behaviour over the surface in different ways.

Jolibois et al. [38] experiment with optimizing the location of the plasma actuator on the surface of a NACA 0015 airfoil, with the purpose of controlling flow separation. This is performed by placing seven plasma actuators at locations ranging from $30 \%$ to $78 \%$ chord of the airfoil with the induced velocity directed in both the opposite and the same direction as the freestream. Each actuator is capable of being activated independently of the other six. Quiescent air testing shows that the actuator is capable of inducing a velocity of $5.5 \mathrm{~m} / \mathrm{s}$.

For the purpose of reattaching the flow to the airfoil Jolibois et al. [38] find that, at an AoA of $15^{\circ}$ the natural point of separation occurs at $45 \%$ chord. The freestream flow is set to Reynolds number of $0.4 \times 10^{6}$, and a velocity of $6 \mathrm{~m} / \mathrm{s}$. PIV measurements show that activation of the plasma actuators located downstream of the natural point of separation are capable of moving the location of separation downstream or partially reattaching the flow. Actuators near or upstream of the point of separation prove to be more capable of reattaching the flow. This is likely due to the plasma actuator adding momentum to the boundary layer where the adverse pressure gradient is lower or at a critical point of inflection. 
With the intent of detaching the flow Jolibois et al. [38] place the airfoil at an AoA of $12^{\circ}$, at which the natural separation point occurs near $74 \%$ of the chord. PIV analysis of the flow field for each of the seven actuators in turn shows that actuators located near the natural point of separation are more capable of separating the flow than those closer to the leading edge. The best separation occurs during the activation of the actuator located at $70 \%$ of the chord just upstream of the natural separation point. This location is where the adverse pressure gradient is at an inflection point. The plasma actuator can remove momentum from the boundary layer to increase the amount of separation present.

He and Corke [9] use plasma actuators as a method of reproducing flaps and slats. A plasma actuator placed at the leading edge of the airfoil such that the induced velocity is in the direction of the freestream, is intended to act as a slat. This works by allowing flow that would typically travel along the lower surface to travel the upper surface and increase the momentum in the suction side boundary layer. A second actuator at the $90 \%$ chord location acts as a flap or aileron by increasing the total lift of the wing throughout all angles of attack. The actuator configuration used is capable of inducing a velocity of $1 \mathrm{~m} / \mathrm{s}$ measured at $9 \mathrm{~mm}$ downstream of the exposed electrode. With activation of the leading edge plasma actuator little difference in lift coefficient is observable below the angle of natural separation. Past the point of natural separation, at $21 \mathrm{~m} / \mathrm{s}$, the leading edge actuator maintained flow attachment as far as $18^{\circ}$ AoA, an increase of $3^{\circ}$ from the un-actuated case. The plasma actuator located at $90 \%$ of the chord showed an increase in lift coefficient 
through all of the examined AoA.

Research performed by Patel et al. [39] compare leading edge plasma actuators, placed such that the induced velocity is in the same direction as the freestream, to typical trailing edge flaps and ailerons. The examined wing is a tactical UCAV 1303 wing, with a $47^{\circ}$ leading edge sweep. This particular wing is designed for a blended wing UAV. Patel et al. examine the effect of plasma actuation at a Reynolds number

of $4.21 \times 10^{5}$. It is proven that leading edge actuators exceed lift enhancement of flaps and ailerons at $5^{\circ}$ of deflection through AoA of $15^{\circ}$ to $24^{\circ}$. The increase of lift coefficient, $C_{L}$, is approximately $\Delta C_{L}=0.1$ at an AoA of $23^{\circ}$. Little to no difference in lift is found between the actuated and baseline cases for a range of AoA between $0^{\circ}$ and $10^{\circ}$. This study shows that plasma actuators are capable of providing an element of control to a wing where three dimensional flows are present. However, the behaviour of this control does not match that of conventional hinged control surfaces.

\subsection{Actuator Operational Freestream Velocity Range}

The ability of DBD plasma actuators to function through a wide range of velocities is important with regards to the functionality of the plasma actuator on aerodynamic vehicles. Aircraft and rockets experience a wide range of speeds throughout flight, and a DBD plasma actuator must match a wide range of freestream velocities to provide diversity to its function. The experiments discussed previously show that 
plasma actuators are capable of manipulating air flow at velocities on the order of $30 \mathrm{~m} / \mathrm{s}$ [9]. This section will explore experiments showing flow control at higher velocities.

Opaits et al. [40] examine the pressure differential across a NACA 0015 airfoil, through a range of velocities and AoA. The experiment proves that flow attachment past the natural separation angle of $15^{\circ}$ is possible up to $75 \mathrm{~m} / \mathrm{s}$, maintaining flow attachment as high as $21^{\circ}$.

Patel et al. [41] use leading edge SDBDs to show that increases in lift can be achieved at velocities as high as $60 \mathrm{~m} / \mathrm{s}$, or a Reynolds number of $1 \times 10^{6}$. It is found that the required voltage to reattach the flow is strongly dependent on the AoA past the stall angle. Results show that the minimum voltage required to attach flow to the airfoil is lower at higher Reynolds numbers on a NACA 0015 airfoil. However, the minimum voltage required to attach flow to a NACA 0006 airfoil increases with Reynolds number. This indicates that the effect of the plasma actuator with respect to Reynolds number is dependent on the shape of airfoil. It is concluded that the minimum voltage required to induce flow reattachment is only weakly dependent on the Reynolds number. 


\subsection{Roll Control Using Plasma Actuators}

Changing the behaviour of the flow over a surface is the basis of control systems in aeronautic vehicles. Plasma actuators are shown to manipulate the behaviour of airflow over a surface. The roll direction is commonly controlled by ailerons on fixed wing aircraft. Plasma actuators mounted on airfoils and flat plates are shown to increase the coefficient of lift. By applying the actuators asymmetrically the span of an air foil or wing a roll moment is induced. This is shown to be equivalent to the use of an aileron for roll control.

Nelson et al. [42] experiment with a UAV wing design (UCAV 1303) and leading edge plasma actuators. It is found that leading edge plasma actuators can create stable roll conditions. The plasma actuators can be switched on and off to induce roll in the vehicle. Through Reynolds numbers between $1.5 \times 10^{5}$ to $4.5 \times 10^{5}$, or velocities of $10 \mathrm{~m} / \mathrm{s}$ to $30 \mathrm{~m} / \mathrm{s}$, an increase in lift coefficient is observed. Calculations showed the level of control achievable by plasma actuation is comparable to that of conventional roll control surfaces, ailerons. Programming the plasma actuators to control the roll over 20 seconds of flight shows a close comparison to a modelled version. Variations in the roll values are observed at higher freestream velocities. It is speculated that this is due to the higher roll moments at these velocities.

In addition to examining lift enhancements, He and Corke uses trailing edge plasma actuators to examine roll control of an aircraft [9]. A DBD plasma actuator is placed on the trailing edge of a NACA 0015 airfoil at 90\% chord length. The AoA 
is increased from $-2^{\circ}$ to $12^{\circ}$, with a freestream velocity of $21 \mathrm{~m} / \mathrm{s}$. The resulting lift force produced by the plasma actuator is compared to the deflection of an aileron from a Navion general aviation aircraft, expressed as $\delta_{a}$. It is found that the plasma actuator produces a similar roll coefficient to a $2.5^{\circ}$ deflection of the aileron. In this case the actuators are placed near the wing tips but only spanning a fraction of the total wing. Assuming that the plasma actuators stretch the entire span of the wing, from wing tip to axis of rotation, the relative deflection angle of the aileron is speculated to increase to $\delta_{a}=9^{\circ}$. It is concluded that applying greater voltages to the plasma actuator can induce greater roll moments on the wing.

Vorobiev et al. [43] also experiment with lift enhancement roll control using trailing edge actuators. The experiment uses a NACA 0009 airfoil at Reynolds numbers of, $2.7 \times 10^{4}<\mathrm{R}_{\mathrm{e}}<1.25 \times 10^{5}$. To induce roll on the airfoil two plasma actuators are mounted on the suction side of the airfoil each independently controllable. Each plasma actuator spans from the wing tip to root for full span control. The actuators operate with a positive saw tooth signal at $2.9 \mathrm{kHz}$ using 65 watts of power each. Activating both plasma actuators creates increases the lift coefficient the airfoil experiences. Examining the effect of Reynolds number with respect to the lift enhancement it is shown that the lift enhancement increases asymptotically to a constant value at higher wind speeds.

Vorobiev et al. examines roll control of the airfoil by activating one of the plasma actuators while the other remains off. The process is repeated with the opposite actuator to induce roll in the reverse direction. The resulting roll moment increases 
asymptotically with increasing Reynolds number, similar to the results found in the lift enhancement. The roll moment converts to a corresponding flap deflection angle at zero AoA using linear airfoil theory. The theoretical flap is located at the actuator location, $75 \%$ chord. The actuators provided a lift equivalent of a flap deflection of $3^{\circ}$ at wind speeds of $2 \mathrm{~m} / \mathrm{s}$ and decreases exponentially to a flap deflection of $0.25^{\circ}$ at $9 \mathrm{~m} / \mathrm{s}$. It is concluded that this decay of equivalent flap angle is due to the asymptotic lift enhancement produced by the actuator relative to a constant lift enhancement from a flap.

\subsection{Relevance}

The relevance of the research described above has a multitude of implications on the research performed here. This effects parameters set within the proposed boundary condition as well as choices in the specific solver and validation methods. The research shown above allows for greater understanding of the capabilities of the DBD plasma actuator and how it may be used within and aerodynamic system. Researchers such as He and Vorobiev [9][43] show that roll control through actuation is in fact possible. The experimentation performed here provide a predictive basis for future testing of plasma actuators during a sounding rocket launch.

The highest tested velocity tested within the literatures is $75 \mathrm{~m} / \mathrm{s}$, [40]. To ensure that the simulations are performed within a reasonable variation of the velocities tested in the literature a velocity range of $90 \mathrm{~m} / \mathrm{s}$ to $10 \mathrm{~m} / \mathrm{s}$ is tested. These velocities 
are below Mach 0.3 at sea level and therefore an incompressible assumption can be made with regards to the simulations. This assumption simplifies the simulations and reduces computational time.

Research by a multitude of authors shows that the induced velocity profile of DBD plasma actuators in quiescent flow has the same general form [21][36][35][38]. This profile consists of a maximum velocity occurring a few millimetres above the surface before returning to zero at the surface. This consistency in velocity profile allows for a simulation of quiescent flow of the boundary condition compared to empirical data to show how effective the proposed boundary condition to represent a DBD plasma actuator, as is shown in later sections. 


\section{Chapter 2}

\section{Computational Theory}

\subsection{OpenFOAM}

Open Source Field Operation and Manipulation (OpenFOAM) computational fluid dynamic (CFD) package is used in this analysis to perform the computations necessary to solve the fluid dynamic equations. OpenFOAM is an open source amalgamation of $\mathrm{C}++$ libraries that make up the solvers and routines within the program. OpenFOAM's collection of solvers can address a wide range of simulations available, including, but not limited to, incompressible flow, compressible flow and reaction dynamics. Paired with these solvers within the OpenFOAM package are an array of utilities to aid in constructing and processing the computational simulations. The utilities used to model the DBD plasma actuator and rocket fin examined here are the meshing tool BlockMesh, and the post processing program ParaView. The architecture of OpenFOAM is expressed broadly in Figure 2.

OpenFOAM version 2.4. $\mathrm{x}$ is used to perform the simulations on a 64 bit Linux 


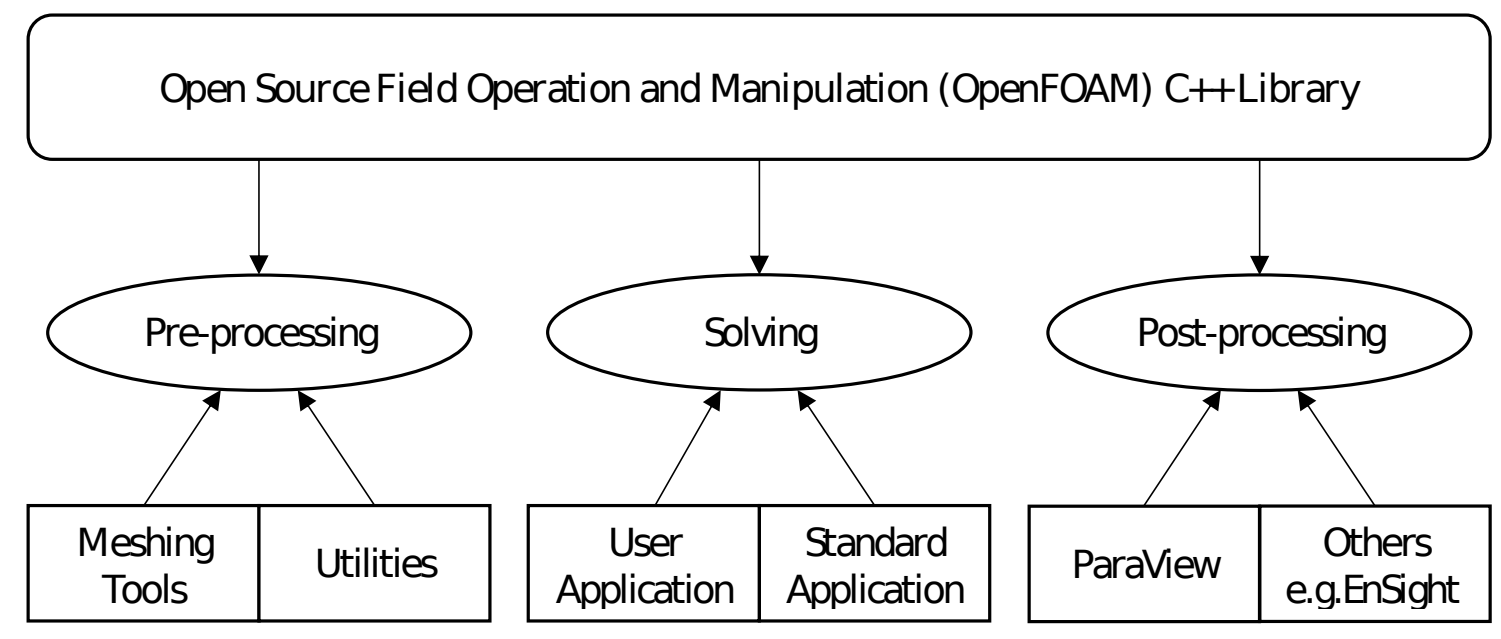

Figure 2: General description of OpenFOAM layout [44]

operating system with two octo-core 3.1 GHz Intel Xenon E5-2687W CPUs fitted with $64 \mathrm{~GB}$ of RAM. The results of the simulations are visualized and analyzed using the post processing software ParaView version 5.3.x.

\subsection{Navier-Stokes Equations}

The Navier-Stokes equations describe the behaviour of viscous fluids. The combination of three fundamental principles that make up the governing equations: conservation of mass, Newton's second law and the conservation of energy. The combination of these three principles in discrete form allows the CFD solver to calculate the properties of the fluid at any point in the flow field.

The conservation of mass can be expressed through the continuity equation. In the partial differential form shown below, the continuity equation shows the sum of the 
change in density over time and the change in mass flux within the domain equates to zero, and therefore mass is conserved.

$$
\frac{\partial \rho}{\partial t}+\frac{\partial \rho u}{\partial x}+\frac{\partial \rho v}{\partial y}+\frac{\partial \rho w}{\partial z}=0
$$

Where, $\rho$ is the density, $t$ is time, $u, v$ and $w$ are the components of the velocity vector.

The momentum equation is based on Newton's second law of motion. Considering all of the forces that may be acting on an element of the fluid, including surface forces (pressure $P$ ), shear stresses (viscosity $\mu$ ) and body forces (external force $f_{x}$ ). The momentum equation includes all of the possible viscous stresses and pressures that can act on a unit of fluid. The momentum equation in the $\mathrm{X}$ direction is expressed in conservation form as,

$$
\begin{array}{r}
\frac{\partial(\rho u)}{\partial t}+\frac{\partial\left(\rho u^{2}\right)}{\partial x}+\frac{\partial(\rho u v)}{\partial y}+\frac{\partial(\rho u w)}{\partial z}=-\frac{\partial P}{\partial x}+\frac{\partial}{\partial x}\left[\lambda\left(\frac{\partial u}{\partial x}+\frac{\partial v}{\partial y}+\frac{\partial w}{\partial z}\right)+2 \mu \frac{\partial u}{\partial x}\right] \\
+\frac{\partial}{\partial y}\left[\mu\left(\frac{\partial u}{\partial y}+\frac{\partial v}{\partial x}\right)\right]+\frac{\partial}{\partial z}\left[\mu\left(\frac{\partial u}{\partial z}+\frac{\partial w}{\partial x}\right)\right]+\rho f_{x}
\end{array}
$$

Similarly, the momentum equation in the Y direction, 


$$
\begin{array}{r}
\frac{\partial(\rho v)}{\partial t}+\frac{\partial(\rho u v)}{\partial x}+\frac{\partial\left(\rho v^{2}\right)}{\partial y}+\frac{\rho v w}{\partial z}=-\frac{\partial P}{\partial y}+\frac{\partial}{\partial y}\left[\lambda\left(\frac{\partial u}{\partial x}+\frac{\partial v}{\partial y}+\frac{\partial w}{\partial z}\right)+2 \mu \frac{\partial v}{\partial y}\right] \\
+\frac{\partial}{\partial x}\left[\mu\left(\frac{\partial v}{\partial x}+\frac{\partial u}{\partial y}\right)\right]+\frac{\partial}{\partial z}\left[\mu\left(\frac{\partial v}{\partial z}+\frac{\partial w}{\partial y}\right)\right]+\rho f_{y}
\end{array}
$$

and the $\mathrm{Z}$ direction,

$$
\begin{array}{r}
\frac{\partial(\rho w)}{\partial t}+\frac{\partial(\rho u w)}{\partial x}+\frac{\partial(\rho v w)}{\partial y}+\frac{\rho w^{2}}{\partial z}=-\frac{\partial P}{\partial z}+\frac{\partial}{\partial z}\left[\lambda\left(\frac{\partial u}{\partial x}+\frac{\partial v}{\partial y}+\frac{\partial w}{\partial z}\right)+2 \mu \frac{\partial w}{\partial z}\right] \\
+\frac{\partial}{\partial x}\left[\mu\left(\frac{\partial w}{\partial x}+\frac{\partial u}{\partial z}\right)\right]+\frac{\partial}{\partial y}\left[\mu\left(\frac{\partial w}{\partial y}+\frac{\partial v}{\partial z}\right)\right]+\rho f_{z}
\end{array}
$$

Where $\lambda$ is the bulk viscosity (or second viscosity) and represents [45]

$$
\lambda=\frac{-2}{3} \mu
$$

The third principle in the triad is the conservation of energy which describes the flow of energy within the fluid. This is made up of the internal energy, $e$, based on the molecular motion, and the kinetic energy due to the motion of the fluid. The equation below describes these forms of energy within the fluid where $E$ is the total energy expressed as $E=e+\frac{U^{2}}{2}$, where $U$ is the magnitude of the velocity vector. The thermal conductivity of the fluid is expressed as $k, T$ is the temperature and $\tau$ is the stress tensor. 


$$
\begin{array}{r}
\frac{\partial(\rho E)}{\partial t}+\frac{\partial(\rho E u)}{\partial x}+\frac{\partial(\rho E v)}{\partial y}+\frac{\partial(\rho E w)}{\partial z}=\rho \dot{q}+\frac{\partial}{\partial x}\left(k \frac{\partial T}{\partial x}\right)+\frac{\partial}{\partial y}\left(k \frac{\partial T}{\partial y}\right)+ \\
\frac{\partial}{\partial z}\left(k \frac{\partial T}{\partial z}\right)-\frac{\partial(u P)}{\partial x}-\frac{\partial(v P)}{\partial y}-\frac{\partial(w P)}{\partial z}+\frac{\partial\left(u \tau_{x x}\right)}{\partial x}+\frac{\partial\left(u \tau_{y x}\right)}{\partial y}+\frac{\partial\left(u \tau_{z x}\right)}{\partial z}+ \\
\frac{\partial\left(v \tau_{x y}\right)}{\partial x}+\frac{\partial\left(v \tau_{y y}\right)}{\partial y}+\frac{\partial\left(v \tau_{z y}\right)}{\partial z}+\frac{\partial\left(w \tau_{x z}\right)}{\partial x}+\frac{\partial\left(w \tau_{y z}\right)}{\partial y}+\frac{\partial\left(w \tau_{z z}\right)}{\partial z}+ \\
\rho\left(f_{x} u+f_{y} v+f_{z} w\right)
\end{array}
$$

The stress tensor $\tau$ is defined below for each element acting on the fluid. As shown here the bulk viscosity parameter is defined with the gradient $\nabla \cdot$ and the velocity vector $\boldsymbol{u}$. This is done here for the purpose of brevity.

$$
\begin{array}{lll}
\tau_{x x}=2 \mu \frac{\partial u}{\partial x}+\lambda \nabla \cdot \boldsymbol{u} & \tau_{y y}=2 \mu \frac{\partial v}{\partial y}+\lambda \nabla \cdot \boldsymbol{u} & \tau_{z z}=2 \mu \frac{\partial w}{\partial z}+\lambda \nabla \cdot \boldsymbol{u} \\
\tau_{x y}=\tau_{y x}=\mu\left(\frac{\partial u}{\partial y}+\frac{\partial v}{\partial x}\right) & \tau_{x z}=\tau_{z x}=\mu\left(\frac{\partial u}{\partial z}+\frac{\partial w}{\partial x}\right) & \tau_{y z}=\tau_{z y}=\mu\left(\frac{\partial v}{\partial z}+\frac{\partial w}{\partial y}\right)
\end{array}
$$

To simulate the flow within the domain a solver must be chosen to work through the above equations to arrive at a solution. This analysis uses the Semi-Implicit Method for Pressure Linked Equations (SIMPLE) solver as the numerical solver. The SIMPLE solver uses finite volume method in an iterative process to calculate the parameters of the flow in each finite volume within the computational domain. The SIMPLE solver assumes steady state incompressible flow, with constant temperature.

The Navier-Stokes equations can be simplified to match with these assumptions. [45]. The assumption of zero change in temperature made in the solver allows for 
the energy equation to be removed completely from the calculations.

The steady state assumption allows for the removal of any time variant parts of the continuity equation and the momentum equations, reducing the continuity equation to,

$$
\frac{\partial \rho u}{\partial x}+\frac{\partial \rho v}{\partial y}+\frac{\partial \rho w}{\partial z}=0
$$

the incompressible assumption provides a constant density throughout the domain simplifying the continuity equation to,

$$
\frac{\partial u}{\partial x}+\frac{\partial v}{\partial y}+\frac{\partial w}{\partial z}=0
$$

Furthermore, the constant viscosity assumption allows for the removal of the gradient terms of viscosity from the momentum equation. With these assumptions, the momentum equation can now be expressed as,

$$
\begin{aligned}
& \rho\left(\frac{\partial\left(u^{2}\right)}{\partial x}+\frac{\partial(u v)}{\partial y}+\frac{\partial(u w)}{\partial z}\right)=-\frac{\partial P}{\partial x}+\mu \frac{\partial}{\partial x}\left[-\frac{2}{3}\left(\frac{\partial u}{\partial x}+\frac{\partial v}{\partial y}+\frac{\partial w}{\partial z}\right)+2 \frac{\partial u}{\partial x}\right] \\
&+\mu \frac{\partial}{\partial y}\left[\left(\frac{\partial u}{\partial y}+\frac{\partial v}{\partial x}\right)\right]+\mu \frac{\partial}{\partial z}\left[\left(\frac{\partial u}{\partial z}+\frac{\partial w}{\partial x}\right)\right]+\rho f_{x}
\end{aligned}
$$

This can be simplified to 


$$
\begin{array}{r}
\rho\left(\frac{\partial\left(u^{2}\right)}{\partial x}+\frac{\partial(u v)}{\partial y}+\frac{\partial(u w)}{\partial z}\right)=-\frac{\partial P}{\partial x}+ \\
\mu \frac{1}{3} \frac{\partial}{\partial x}\left(\frac{\partial u}{\partial x}+\frac{\partial v}{\partial y}+\frac{\partial w}{\partial z}\right)+\mu\left(\frac{\partial^{2} u}{\partial x^{2}}+\frac{\partial^{2} u}{\partial y^{2}}+\frac{\partial^{2} u}{\partial z^{2}}\right)+\rho f_{x}
\end{array}
$$

As proved in the continuity equation, Equation 8, the divergence of velocity is equal to zero. Therefore, the momentum equation can be simplified to the final equations used in the SIMPLE solver. The final equations expressed in X Y and Z directions are:

$$
\begin{aligned}
\rho\left(\frac{\partial\left(u^{2}\right)}{\partial x}+\frac{\partial(u v)}{\partial y}+\frac{\partial(u w)}{\partial z}\right) & =-\frac{\partial P}{\partial x}+\mu\left(\frac{\partial^{2} u}{\partial x^{2}}+\frac{\partial^{2} u}{\partial y^{2}}+\frac{\partial^{2} u}{\partial z^{2}}\right) \\
\rho\left(\frac{\partial(u v)}{\partial x}+\frac{\partial\left(v^{2}\right)}{\partial y}+\frac{\partial(v w)}{\partial z}\right) & =-\frac{\partial P}{\partial y}+\mu\left(\frac{\partial^{2} v}{\partial x^{2}}+\frac{\partial^{2} v}{\partial y^{2}}+\frac{\partial^{2} v}{\partial z^{2}}\right) \\
\rho\left(\frac{\partial(u w)}{\partial x}+\frac{\partial(v w)}{\partial y}+\frac{\partial\left(w^{2}\right)}{\partial z}\right) & =-\frac{\partial P}{\partial z}+\mu\left(\frac{\partial^{2} w}{\partial x^{2}}+\frac{\partial^{2} w}{\partial y^{2}}+\frac{\partial^{2} w}{\partial z^{2}}\right)
\end{aligned}
$$

Without the presence of an external force acting on the fluid, $f=0$ and therefore it is removed from the equations.

The SIMPLE solver is a Reynolds-averaged Navier-Stokes (RANS) solver with an incompressible flow assumption and viscous effects. This method of solving uses a statistically steady flow where each velocity component can be represented as the sum of a time averaged value and a fluctuation about that average value. 
This depicts the velocity at any one time as the average component $\bar{u}$ and the instantaneous component $u^{\prime}$ to form the equation,

$$
u=\bar{u}+u^{\prime}
$$

This introduces more variables to the system that need to be solved. To solve for these new unknowns a turbulence model is added. This analysis uses the k-omega shear stress transport (SST) model to address these new variables. In the following equations the components of velocity $u, v$ and $w$ are representative of the time averaged and instantaneous flow velocities.

The Navier-Stokes equations are discretized using a Gaussian finite volume integration scheme such that the equations can be used within a finite computational domain. The Gaussian integration scheme interpolates from the centre of the cell to the cell face. Several schemes are present in the complete discretized formulas gradient and Laplacian, $\nabla^{2}$, sections of the simplified Navier-Stokes equations use a second order central differencing scheme. For sections of divergence, $\nabla \cdot$, of the equations this analysis uses a first order upwind discretization scheme.

The first order upwind scheme used within this analysis for sections of divergence provides a discretization shown in Equation 15. This method is used for the velocity divergence section of the momentum equation. 


$$
\frac{\partial}{\partial x} f(x)=\frac{f(x)-f(x-\Delta x)}{\Delta x}
$$

The gradient and Laplacian sections of the equations, including the viscous component and pressure component of the momentum equations, use a second order central differencing method that follow the structure of Equation 16 and Equation 17. The discretization schemes used here equations are taken from Leveque [46] for the discretization of a uniform grid.

$$
\begin{gathered}
\frac{\partial f(x)}{\partial x}=\frac{f(x+\Delta x)-f(x-\Delta x)}{2 \Delta x} \\
\frac{\partial^{2} f(x)}{\partial x^{2}}=\frac{f(x+\Delta x)-2 f(x)+f(x-\Delta x)}{(\Delta x)^{2}}
\end{gathered}
$$

These discretization methods are used in the SIMPLE solver which is explored in detail here. The equations shown here solve for a two-dimensional domain. The finite grid notations are depicted with I and J, according to Figure 3 below. The datum location is placed in the middle and is denoted with the coordinates of I,J. The neighbouring black points are depicted with I and J plus or minus the difference between the specific location and the datum.

The white points depict the staggered grid. The staggered grid is implemented to prevent the smoothing of fluctuating pressure fields that may occur within the flow. Variables that consist of a vector, such as velocity, are measured at the staggered grid points designated $\mathrm{i}$ and $\mathrm{j}$. While scalar values (such as pressure and viscosity) 
are measured at the points designated I and J.

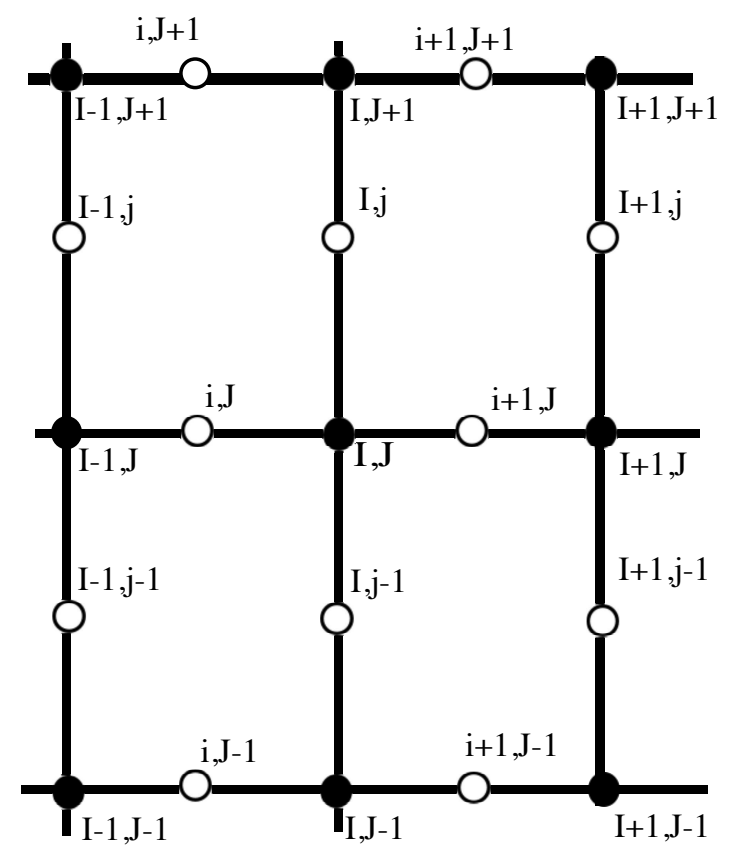

Figure 3: A simple annotation of the grid and the location of the points used in the following equations.

With this grid and the discretization methods mentioned above the momentum equation can be discretized in to the following form,

$$
\begin{array}{r}
u_{i, J}\left(\rho u_{i, J}+\frac{\mu_{I, J}}{\delta x}\right)=u_{i-1, J}\left(\rho u_{i-1, J}+\frac{\mu_{I, J}}{\delta x}\right)-v_{i, J}\left(\rho u_{i, J}\right) \\
+v_{i, J-1}\left(\rho u_{i, J-1}\right)+u_{i+1, J}\left(\frac{\mu_{I, J}}{\delta x}\right)-u_{i, J}\left(\frac{\mu_{I, J}}{\delta x}\right)+u_{i, J-1}\left(\frac{\mu_{I, J}}{\delta y}\right) \\
-2 u_{i, J}\left(\frac{\mu_{I, J}}{\delta y}\right)+u_{i, J+1}\left(\frac{\mu_{I, J}}{\delta y}\right)+\left(P_{I-1, J}-P_{I, J}\right) A_{u}
\end{array}
$$


Where $A_{u}$ is the area of the cell face. A similar formula is used to find the velocity in the $\mathrm{Y}$ direction.

$$
\begin{array}{r}
v_{I, j}\left(\rho v_{I, j}+\frac{\mu_{I, J}}{\delta y}\right)=v_{I, j-1}\left(\rho v_{I, j-1}+\frac{\mu_{I, J}}{\delta y}\right)-u_{I, j}\left(\rho v_{I, j}\right) \\
+u_{I-1, j}\left(\rho v_{I-1, j}\right)+v_{I, j+1}\left(\frac{\mu_{I, J}}{\delta y}\right)-v_{I, j}\left(\frac{\mu_{I, J}}{\delta y}\right)+v_{I-1, j}\left(\frac{\mu_{I, J}}{\delta x}\right) \\
-2 v_{I, j}\left(\frac{\mu_{I, J}}{\delta x}\right)+v_{I+1, j}\left(\frac{\mu_{I, J}}{\delta x}\right)+\left(P_{I, J-1}-P_{I, J}\right) A_{u}
\end{array}
$$

These equations are simplified further into the form used by Versteeg [47]. Where the velocity and viscosity fields are combine into a variable $a$, and is representative of,

$$
a=\rho u+\frac{\mu}{\delta x}
$$

The momentum equation in the $\mathrm{X}$ and $\mathrm{Y}$ directions can then be expressed as

$$
\begin{aligned}
& a_{i, J} u_{i, J}=\sum a_{n, b} u_{n, b}+\left(P_{I-1, J}-P_{I, J}\right) A_{i, J} \\
& a_{I, j} v_{I, j}=\sum a_{n, b} v_{n, b}+\left(P_{I, J-1}-P_{I, J}\right) A_{I, j}
\end{aligned}
$$

These discretized momentum equations are implemented within the SIMPLE algorithm to solve for the pressure and velocity fields within the computational domain. 
The first step of the SIMPLE process begins with the initial pressure field. The initial estimated values are designated with an $*$. In the case of the pressure field it is expressed as $P^{*}$. This pressure field is used in the momentum equations, displayed below, to estimate the velocity field. The values of $u$ and $v$ have been found using $P^{*}$, and therefore they are also designated by $*$. The $\mathrm{X}$ and $\mathrm{Y}$ momentum equations are then expressed as,

$$
\begin{aligned}
& a_{i, J} u_{i, J}^{*}=\sum a_{n, b} u_{n, b}^{*}+\left(P_{I-1, J}^{*}-P_{I, J}^{*}\right) A_{i, J} \\
& a_{I, j} v_{I, j}^{*}=\sum a_{n, b} v_{n, b}^{*}+\left(P_{I, J-1}^{*}-P_{I, J}^{*}\right) A_{I, j}
\end{aligned}
$$

The values of $\left(P^{*}\right),\left(\rho u^{*}\right)$ and $\left(\rho v^{*}\right)$ are initial guesses that may not satisfy the continuity equation. A pressure correction factor is used as the difference between the correct values and the guessed values for each iteration, shown in the equations below. As the difference between these values decreases approaching convergence the correction factor approaches zero.

$$
P=P^{*}+P^{\prime} \quad u=u^{*}+u^{\prime} \quad v=v^{*}+v^{\prime}
$$

The correction factor values designated with the symbol '. These are then introduced into the momentum equations, at steady state to produce the corrected momentum equations in the $\mathrm{X}$ and $\mathrm{Y}$ directions, 


$$
\begin{aligned}
& a_{i, J}\left(u_{i, J}-u_{i, J}^{*}\right)=\sum a_{n, b}\left(u_{n, b}-u_{n, b}^{*}\right)+\left[\left(P_{I-1, J}-P_{I-1, J}^{*}\right)-\left(P_{I, J}-P_{I, J}^{*}\right)\right] A_{i, J} \\
& a_{I, j}\left(v_{I, j}-v_{I, j}^{*}\right)=\sum a_{n, b}\left(v_{n, b}-v_{n, b}^{*}\right)+\left[\left(P_{I, J-1}-P_{I, J-1}^{*}\right)-\left(P_{I, J}-P_{I, J}^{*}\right)\right] A_{I, j}
\end{aligned}
$$

This is rewritten as,

$$
\begin{aligned}
& a_{i, J} u_{i, J}^{\prime}=\sum a_{n, b} u_{n, b}^{\prime}+\left(P_{I-1, J}^{\prime}-P_{I, J}^{\prime}\right) A_{i, J} \\
& a_{I, j} v_{I, j}^{\prime}=\sum a_{n, b} v_{n, b}^{\prime}+\left(P_{I, J-1}^{\prime}-P_{I, J}^{\prime}\right) A_{I, j}
\end{aligned}
$$

It is then assumed that within the correction equations the viscosity term can be ignored. This is the approximation of the SIMPLE algorithm around which the process hinges. Furthermore, the orthogonal components of velocity are ignored producing the equations,

$$
\begin{aligned}
& u_{i, J}^{\prime}=\frac{A_{i, J}}{a_{i, J}}\left(P_{I-1, J}^{\prime}-P_{I, J}^{\prime}\right) \\
& v_{I, j}^{\prime}=\frac{A_{I, j}}{a_{I, j}}\left(P_{I, J-1}^{\prime}-P_{I, J}^{\prime}\right)
\end{aligned}
$$

These equations can be expressed with respect to $u$ and $v$ through the correction factor equations, 


$$
\begin{aligned}
& u_{i, J}=u_{i, J}^{*}+\frac{A_{i, J}}{a_{i, J}}\left(P_{I-1, J}^{\prime}-P_{I, J}^{\prime}\right) \\
& v_{I, j}=v_{I, j}^{*}+\frac{A_{I, j}}{a_{I, j}}\left(P_{I, J-1}^{\prime}-P_{I, J}^{\prime}\right)
\end{aligned}
$$

This same process is performed with $u_{i+1, J}$ and $v_{I, j+1}$. Later equations are simplified by allowing the quotient of area $A$, and the coefficient $a$ to a single variable, shown in the following equation.

$$
d_{i+1, J}=\frac{A_{i+1, J}}{a_{i+1, J}} \quad d_{I, j+1}=\frac{A_{I, j+1}}{a_{I, j+1}}
$$

The velocity field within the computational domain must also satisfy the continuity equation. Discretized in the following method,

$$
\left[\left(\rho u_{i+1, J} A_{i+1, J}\right)-\left(\rho u_{i, J} A_{i, J}\right)\right]+\left[\left(\rho v_{I, j+1} A_{I, j+1}\right)-\left(\rho v_{I, j} A_{I, j}\right)\right]=0
$$

Substituting Equations 31 and 32 into the continuity equation provides Equation 33 ,

$$
\begin{gathered}
{\left[\rho A_{i+1, J}\left(u_{i+1, J}^{*}+d_{i+1, J}\left(P_{I, J}^{\prime}-P_{I+1, J}\right)\right)-\rho A_{i, J}\left(u_{i, J}^{*}+d_{i, J}\left(P_{I-1, J}^{\prime}-P_{I, J}^{\prime}\right)\right)\right]+} \\
{\left[\rho A_{I, J+1}\left(v_{I, J+1}^{*}+d_{I, j+1}\left(P_{I, J}^{\prime}-P_{I, J+1}^{\prime}\right)\right)-\rho A_{I, j}\left(v_{I, j}^{*}+d_{i, J}\left(P_{I, J-1}^{\prime}-P_{I, J}^{\prime}\right)\right)\right]=0}
\end{gathered}
$$

This can be rearranged to produce Equation 35, 


$$
\begin{array}{r}
{\left[\rho(d A)_{i+1, J}+\rho(d A)_{i, J}+\rho(d A)_{I, j+1}+\rho(d A)_{I, j+1}\right] P_{I, J}^{\prime}=} \\
\rho(d A)_{i+1, J} P_{I+1, J}^{\prime}+\rho(d A)_{i, J} P_{I-1, J}^{\prime}+\rho(d A)_{I, j+1} P_{I, J+1}+\rho(d A)_{I, j} P_{I, J-1}^{\prime} \\
+\left[\rho\left(u^{*} A\right)_{i, J}-\rho\left(u^{*} A\right)_{i+1, J}+\rho\left(v^{*} A\right)_{I, j}-\rho\left(v^{*} A\right)_{I, j+1}\right]
\end{array}
$$

It can be noted that the final terms of this equation are the continuity equation. Thus, the pressure correction factor is driven by the errors inherent to the continuity equation. Furthermore, it can also be noted that this equation is elliptical in nature [45]. This is due to the ability of a pressure variance propagating throughout the entirety of the flow. This also can lead to instabilities that can induce divergence.

The new pressure field for the domain, $P$, is calculated using the sum of the guessed pressure field, $P^{*}$, and the calculated pressure correction factor, $P^{\prime}$. The velocity fields are corrected with Equations 31 and 32. When convergence is reached the value of $P^{\prime}$ will be approximately equal to zero providing the equations $P^{*}=P$, $u^{*}=u$ and $v^{*}=v$.

To promote convergence of the pressure correction factor, relaxation factors are placed on the corrector step of the process for pressure and velocity. This changes the pressure corrector formula to,

$$
P=\left(P^{*}\right)+\beta_{p} P^{\prime}
$$


where beta is $0<\beta_{p} \leq 1$. Lower values of $\beta_{p}$ increase the stability of the solution and deter divergence while higher values increase the chance of the solution diverging. However, lower values of beta require more iterations to arrive at a converged solution, increasing the computational time. A value of $\beta_{p}=0.4$ is used for these simulations.

\subsection{Turbulence Modelling}

Turbulence is implemented to the governing equations through the addition of a time varying component of velocity to the momentum equation. The addition of these time varying components increases the number of unknowns to the system. The k- $w$ SST model introduces two equations to calculate these new terms. The two equations govern the turbulent kinetic energy, $k$, the kinetic energy due to instantaneous velocity variation from the time averaged velocity of the flow, and $\omega$, the rate at which the turbulent eddies dissipate.

The k- $\omega$ SST model is chosen for its ability to capture near wall turbulence and flow separation better than that of the $\mathrm{k}-\varepsilon$ model, while saving on computational power relative to a large eddy simulation, detached eddy simulation, or direct numerical simulation methods.

The k- $\omega$ SST model is a two-equation turbulence model. The equations are based on the linear eddy viscosity assumption made by Boussinesq. The SST is added to 
the k- $\omega$ model by F.R Menter [48]. The addition of a cross-diffusion term in the $\omega$ equation allows the model to be less dependent on the free stream shear. The equations shown below are found to fit specific scenarios such as adverse pressure gradients closer to the empirical data than the baseline $\mathrm{k}-\boldsymbol{\omega}$ model.

Equation 37, the first of the two-equation model, uses parameters within the flow field to calculate the specific turbulent kinetic energy field designated by the variable $k$.

$$
\frac{\partial \rho k}{\partial t}+u \frac{\partial \rho k}{\partial x}+v \frac{\partial \rho k}{\partial y}=\mu \frac{\partial u}{\partial y}\left(\frac{\partial u}{\partial y}+\frac{\partial v}{\partial x}\right)-\beta^{*} \rho \omega k+\frac{\partial}{\partial y}\left[\left(\mu+\sigma_{k} \mu_{t}\right) \frac{\partial k}{\partial y}\right]
$$

where $\mu$ is the viscosity of the fluid and $\mu_{t}$ is the dynamic turbulent eddy viscosity. The second equation, Equation 38, provides the turbulent dissipation, $\omega$. These parameters provide a model of the turbulence that occurs within the computational domain.

$$
\begin{array}{r}
\frac{\partial \rho \omega}{\partial t}+u \frac{\partial \rho \omega}{\partial \omega}+v \frac{\partial \rho \omega}{\partial y}=\mu \frac{\gamma}{\nu_{t}} \frac{\partial u}{\partial y}\left(\frac{\partial u}{\partial y}+\frac{\partial v}{\partial x}\right)-\beta \rho \omega^{2}+ \\
\frac{\partial}{\partial y}\left[\left(\mu+\sigma_{\omega} \mu_{t}\right) \frac{\partial \omega}{\partial y}\right]+2\left(1-F_{2}\right) \frac{\rho \sigma_{\omega 2}}{\omega} \frac{\partial k}{\partial y} \frac{\partial \omega}{\partial y}
\end{array}
$$

These two equations paired with the calculation relating $k$ and $\omega$ to the kinematic 
turbulent eddy viscosity, $\nu_{t}$, provide the equations necessary to calculate all the unknown variables in the system.

$$
\nu_{t}=\frac{k}{\omega}
$$

The SST version of the k- $\omega$ model uses a blending function, $F_{2}$. The function takes advantage of the k- $\omega$ model's superiority at near-wall turbulence characteristics

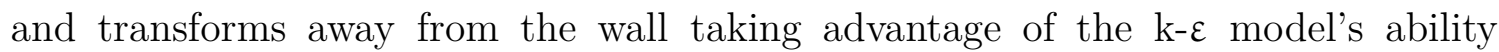
to calculate the turbulence in free shear layers creating a double layering of the turbulence model with respect to height away from the wall. This provides a blend of the k- $\omega$ model in near wall scenarios while allowing for the $\mathrm{k}-\varepsilon$ model to work in the free shear regions.

$$
F_{2}=\tanh \left(\max \left(2 \frac{\sqrt{k}}{0.09 \omega y} ; \frac{500 \nu}{y^{2} \omega}\right)^{2}\right)
$$

The constants of $a, \beta, \beta *, \sigma_{k}, \sigma_{\omega}, \kappa$, and $\gamma$ are used within the k- $\omega$ SST algorithm. These are held constant throughout all the simulations according to the suggested values made by F.R Menter [48]

$$
\begin{array}{crr}
a=0.31 \quad \beta=0.075 & \beta *=0.09 & \kappa=0.41 \\
\sigma_{k}=0.85 & \sigma_{\omega}=0.5 & \gamma=\frac{\beta}{\beta *}-\frac{\sigma_{\omega} \kappa^{2}}{\sqrt{\beta *}}
\end{array}
$$




\section{Chapter 3}

\section{Grid Refinement Study}

\subsection{Two Dimensional Computational Domain}

The rocket fin examined in this study is modelled using the dimensions shown in Figure 4. This drawing is representative of the dimensions of the as-built fin provided by UNB. The two dimensional simulations match the dimensions at the fin tip, thus reducing the overall length of the computational domain.

The two dimensional domain represents the fin as a flat plate of length equal to the length of the fin tip, $14 \mathrm{~cm}$, ignoring the chamfer on the leading edge. The inlet boundary is placed one chord length ahead of the fin and holds the pressure (zero

relative pressure) and the velocity constant. The outlet boundary is located one chord length downstream of the fin's trailing edge. The outlet holds a gradient of zero between the final cell layer and the neighbouring cell layer for both pressure and velocity. The surface of the fin is considered no slip, with exception to the plasma actuator boundary condition discussed below. 


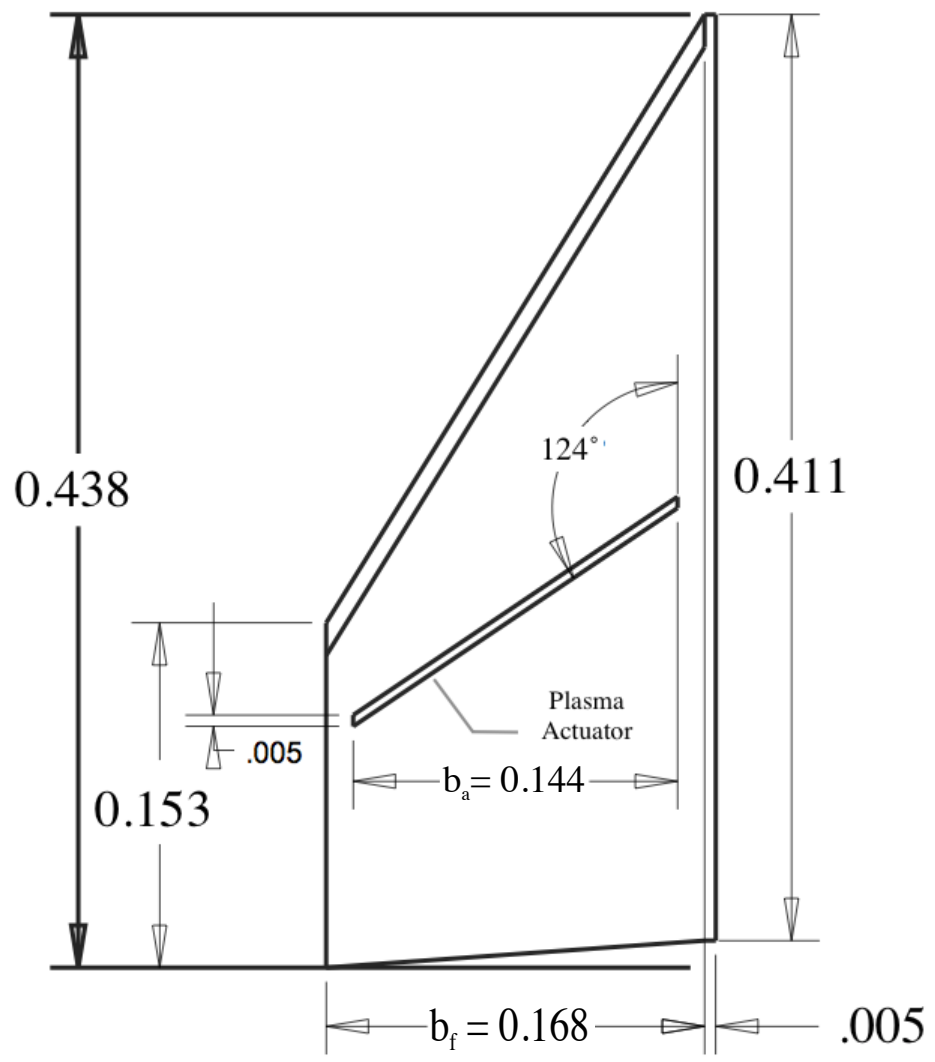

Figure 4: The as-built sounding rocket fin, dimensions in meters. The location of the encapsulated electrode of the plasma actuator is noted.

Due to the flat plate assumption, the computational domain can consist of orthogonal cells made using OpenFOAM's BlockMesh tool, creating a fine mesh of orthogonal cells with user input cell expansion ratios. The use of the block mesh tool saves time in the meshing process while eliminating the possibility of skewed cells.

Previous research shows that a plasma actuator can produce induced velocity 
profiles through a range of maximum velocities measured at $2 \mathrm{~mm}$ to $5 \mathrm{~mm}$ from the surface on which it is mounted [49]. To recreate the induced velocity profile within the simulation, the plasma actuator is represented as a $5 \mathrm{~mm}$ long portion of the fin where the no slip condition is replaced with a tangential surface velocity of $5 \mathrm{~m} / \mathrm{s}$ in the upstream direction. The velocity placed on the surface transfers momentum to the fluid above the surface through viscous effects.

The boundary condition is a simplistic representation of a plasma actuator. This method reduces the computational cost of the simulation and can be easily implemented within a simulation. The plasma actuator is entered as a velocity boundary condition rather than an addition of parameters to the equations within the solver as was done by Shyy [50].

The upper boundary of the computational domain is placed approximately ten boundary layer thicknesses above the fin to prevent the upper boundary from having an effect on the resulting boundary layer produced. The boundary layer thickness is approximated with the assumption of incompressible flow over a flat plate using Equation 41 [45]. This equation also ignores the transition from laminar to turbulent assuming the entirety of the boundary layer is turbulent [51].

$$
\delta=\frac{0.37 x}{R_{e}^{1 / 5}}
$$

Where $\delta$ is the thickness of the boundary layer, $x$ is the location on the plate, and $R_{e}$ is the Reynolds number. At $20 \mathrm{~m} / \mathrm{s}$ the Reynolds number is approximately 
$1.8 \times 10^{5}$. The location of the largest boundary layer is at the trailing edge of the fin when $x$ is equal to $14 \mathrm{~cm}$. The boundary layer thickness at this location is found to be $0.46 \mathrm{~cm}$. The upper boundary of the mesh is therefore placed at $5 \mathrm{~cm}$ above the fin.

An adverse pressure gradient within the boundary layer, due to the simulated plasma actuator is expected. Therefore, wall functions are not used within the simulations. With the lack of wall functions, the first grid point must be within the lower regions of the boundary layer. Therefore, the first grid point above the surface is set such that the $\mathrm{y}+$ value is less than 1.0. The $Y^{+}$value is the non-dimensional distance perpendicular from the surface to the first node of the mesh. The resulting mesh consists of 420,000 cells making up the domain.

The resulting domain has too fine a mesh to discern the individual cells in a diagram. A courser mesh with the same parameters as those used in the simulations is shown in Figure 5 for the purpose of displaying the dimensions of the domain and the method of distribution of the cells within that domain. The gradients in the mesh structure are visible with finer mesh located on the surface of the fin increasing to coarser mesh away from the fin. Further gradients are observed approaching the leading edge located at $X=0$ and following the trailing edge at $X=14 \mathrm{~cm}$.

Figure 5 also shows the bottom of the domain indicating the locations of the fin, the free space and the actuator shown here at $50 \%$ chord within the computational domain. The free space is defined as the space between the inlet of the computational domain and leading edge of the fin as well as the space from the trailing edge of 


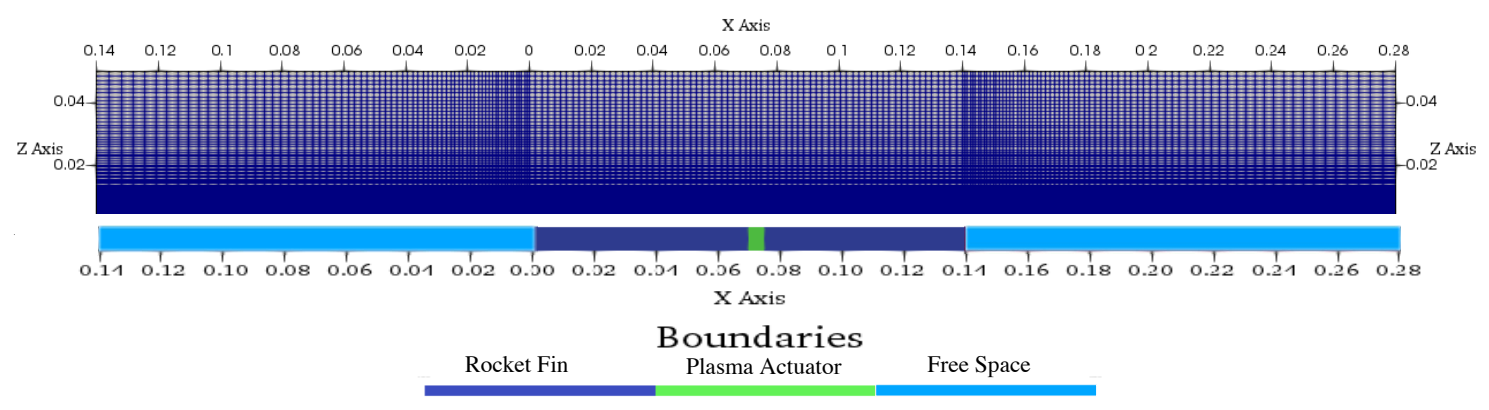

Figure 5: The computational domain with the coarse mesh, displaying the bottom of the domain with the fin and plasma actuator marked

the fin to the outlet of the computational domain. The location of the actuator is fixed within each individual simulation, however the location can be varied between simulations. The effects of the actuator on the pressure field at different locations on the fin is examined in a later section.

Table 1: Boundary conditions for computational domain

\begin{tabular}{|c|c|c|c|c|}
\hline Boundary & Velocity & Pressure & $\begin{array}{c}\text { Turbulent } \\
\text { Kinetic Energy }\end{array}$ & $\begin{array}{c}\text { Turbulent Eddy } \\
\text { Dissipation }\end{array}$ \\
\hline \hline Inlet & Inlet Outlet & Constant & Constant & Constant \\
\hline Outlet & Zero Gradient & Zero Gradient & Inlet Outlet & Inlet Outlet \\
\hline Free Space & Slip Wall & Zero Gradient & Zero Gradient & Zero Gradient \\
\hline Fin & No Slip Wall & Zero Gradient & Zero Gradient & Zero Gradient \\
\hline Actuator & Wall $-5 \mathrm{~m} / \mathrm{s}$ & Zero Gradient & Zero Gradient & Zero Gradient \\
\hline Front \& Back & Empty & Empty & Empty & Empty \\
\hline
\end{tabular}

OpenFOAM requires a definition on the third dimension on a two dimensional domain. This requirement is the origin of the 'Front and Back' boundary. These boundaries are set to the designation of 'Empty' to indicate to OpenFOAM that 
this is a two dimensional simulation and these boundaries represent the ignored third dimension.

\subsection{Verification}

To verify the calculations performed within the OpenFOAM CFD package the boundary layer profile from simulations used within the results chapter of this analysis are compared to theoretical solutions for the boundary layer. A boundary layer profile from a $20 \mathrm{~m} / \mathrm{s}$ simulation with laminar flow is compared to the Blasius solution. While several boundary layer profiles where turbulent flow is expected from a $70 \mathrm{~m} / \mathrm{s}$ simulation are compared to the equation developed by Van Driest. The velocity profiles of both the mathematical and simulation velocity profiles are compared to the mathematical models by way of the shape factor.

\subsubsection{Blasius Comparison}

The boundary layer profiles, both taken from the control case, without the addition of a simulated actuator boundary condition, and a case without the implementation of a turbulence model are compared to that of the Blasius solution. The Blasius solution mathematically describes the exact boundary layer profile on a flat plate parallel to a uniform flow. The Blasius boundary layer is represented by a system of equations shown both by Schetz [51] and White [52]. The equations are solved iteratively using a shooting method to create a velocity profile. 
The perpendicular distance away from the surface is calculated as,

$$
y=\eta \sqrt{\frac{2 \nu x}{U}}
$$

where $\eta$ is the Blasius self similar variable that is non-dimensional. The velocity profile is expressed as the derivative of a function of the self similar variable.

$$
\frac{u}{U}=f^{\prime}(\eta)
$$

This is derived from the definition of the independent variable $\eta$ being a function of $\mathrm{x}$ and $\mathrm{y}$ such that, $u(x, y)=u[\eta(x, y)]$.

The stream function is representative of the stream lines of the fluid (curves which at any fixed moment are tangential to the velocity field) is expressed as a function of the self similar variable, where $\nu$ is the kinematic viscosity of the fluid,

$$
\psi=\sqrt{2 \nu U x} f(\eta)
$$

Differentiating $\psi$ with respect to $\mathrm{x}$ provides the vertical component of velocity $v$ is also expressed in terms of $\eta$, 


$$
v=\sqrt{\frac{\nu U}{2 x}}\left(\eta f^{\prime}(\eta)-f(\eta)\right)
$$

These equations produce the final equation of,

$$
f^{\prime \prime \prime}(\eta)+f(\eta) f^{\prime \prime}(\eta)=0
$$

The fin is considered to have zero slip producing the boundary conditions of $f(0)=f^{\prime}(0)=0$ and the free stream to have a constant velocity $U$ producing the boundary condition $f^{\prime}(\infty)=1$. Naturally examining this parameter to infinity is computationally impossible and therefore a sufficiently large value is used. It is expected that the boundary layer reach $99 \%$ of the free-stream velocity at $\eta \approx 5$, however the functions are examined up to a level of $\eta=10$.

Shooting method is used to solve the equations iteratively to meet the boundary conditions. This method requires two initial guesses at the value of $f^{\prime \prime}(0)$. For this analysis the initial guesses are set to guess $(1)=1$ and $\operatorname{guess}(2)=0.3$. Three arrays are constructed consisting of zeros representing $f(\eta), f^{\prime}(\eta)$ and $f^{\prime \prime}(\eta)$. The first guess is set as a boundary condition at $i=1$ (where $i$ is the index within the array), in $f^{\prime \prime}(\eta)$. The Equations 47 and 48 are created using Euler's method of discretization while Equation 49 is a derivation of backward finite difference method.

$$
f(i)=f(i-1)+f^{\prime}(i-1) \Delta \eta
$$




$$
\begin{gathered}
f^{\prime}(i)=f^{\prime}(i-1)+f^{\prime \prime}(i-1) \Delta \eta \\
f^{\prime \prime}(i)=\frac{f^{\prime}(i-1)-f^{\prime}(i)}{\Delta \eta}
\end{gathered}
$$

These equations are set in the iterative solver where the second guess is input into the boundary condition within Equation 49 and the equations are solved. The guess at the boundary layer of $f^{\prime \prime}(0)$ is updated with each iteration using Equation 50

$$
f^{\prime \prime}(0)^{k+1}=f^{\prime \prime}(0)^{k}-\left(f^{\prime}(\infty)^{k}-1\right) \frac{f^{\prime \prime}(0)^{k}-f^{\prime \prime}(0)^{k-1}}{f^{\prime}(\infty)^{k}-f^{\prime}(\infty)^{k-1}}
$$

In which $k$ represents the number of the iteration. These equations are solved until the value of $f^{\prime}(\infty) \approx 1$. In this analysis the tolerance set to this condition is $1 \times 10^{-8}$. Using this method the solution converges in nine iterations.

The boundary layer profile is calculated at a location $2 \mathrm{~cm}$ from the leading edge of the fin. At this same location, the boundary layer profile is taken from a simulation for comparison.

The Blasius solution assumes that the flow is incompressible and laminar. The SIMPLE solver used in this analysis also uses incompressible flow, however, one of the simulations includes the k-omegaSST turbulence model. With the addition of the turbulence model some variation between this boundary layer profile and the Blasius solution can be expected. The simulation without a turbulence model implemented can be expected to have a close fit to the Blasius solution. 
With a free stream velocity of $20 \mathrm{~m} / \mathrm{s}$, the Reynolds number at a location $2 \mathrm{~cm}$ from the leading edge is found to be approximately $2.5 \times 10^{4}$. The equation below describes the distance $\mathrm{y}$ from the surface of the fin with respect to the velocity within the boundary layer.

This equation is rearranged to find the normalized velocity with respect to the distance, $y$ away from the wall,

$$
\frac{u}{U}=\frac{(y \eta)^{2}}{x}
$$

The y values are then normalized by dividing by the total thickness of the boundary layer at $2 \mathrm{~cm}$ from the leading edge. The boundary layer thickness is calculated using [51],

$$
\delta=\frac{5.0 x}{\sqrt{R e}}
$$

The Blasius solution for the normalized velocity profile can then be graphed as a function of the normalized $\mathrm{y}$-values, $y / \delta$, providing the velocity profile of the boundary layer at $2 \mathrm{~cm}$ from the leading edge.

The simulation data are measured from the surface to the height of the boundary layer. The thickness of the boundary layer at the measured location is considered as the point where the velocity reaches $99 \%$ of the free stream velocity. The collected data from the simulation is then similarly non-dimensionalized. The velocity is 
divided by the free stream velocity of $20 \mathrm{~m} / \mathrm{s}$, and the distance from the surface is divided by the measured boundary layer thickness. These data sets are compared graphically to the Blasius solution in Figure 6.

The boundary layer profiles compared to that of the Blasius solution provide a validation of the correctness of the two-dimensional simulations. A two-dimensional simulation with the convergence residual set to $10^{-4}$ as well as a simulation with the turbulence model turned off leaving fully laminar flow, are shown in Figure 6.

The simulation with the turbulence model implemented is shown to have higher velocities close to the surface of the plate. The cause of this is due to the presence of turbulent kinetic energy throughout the computational domain. The laminar condition implemented within the same domain is shown to have higher velocities throughout its profile, but matches closely to the same shape profile as the Blasius solution.

The boundary layers are also examined on a basis of the shape factor calculated from the quotient of the displacement thickness and the momentum thickness.

$$
H=\frac{\delta^{*}}{\theta}
$$

Where the displacement thickness for incompressible flow is calculated using, 


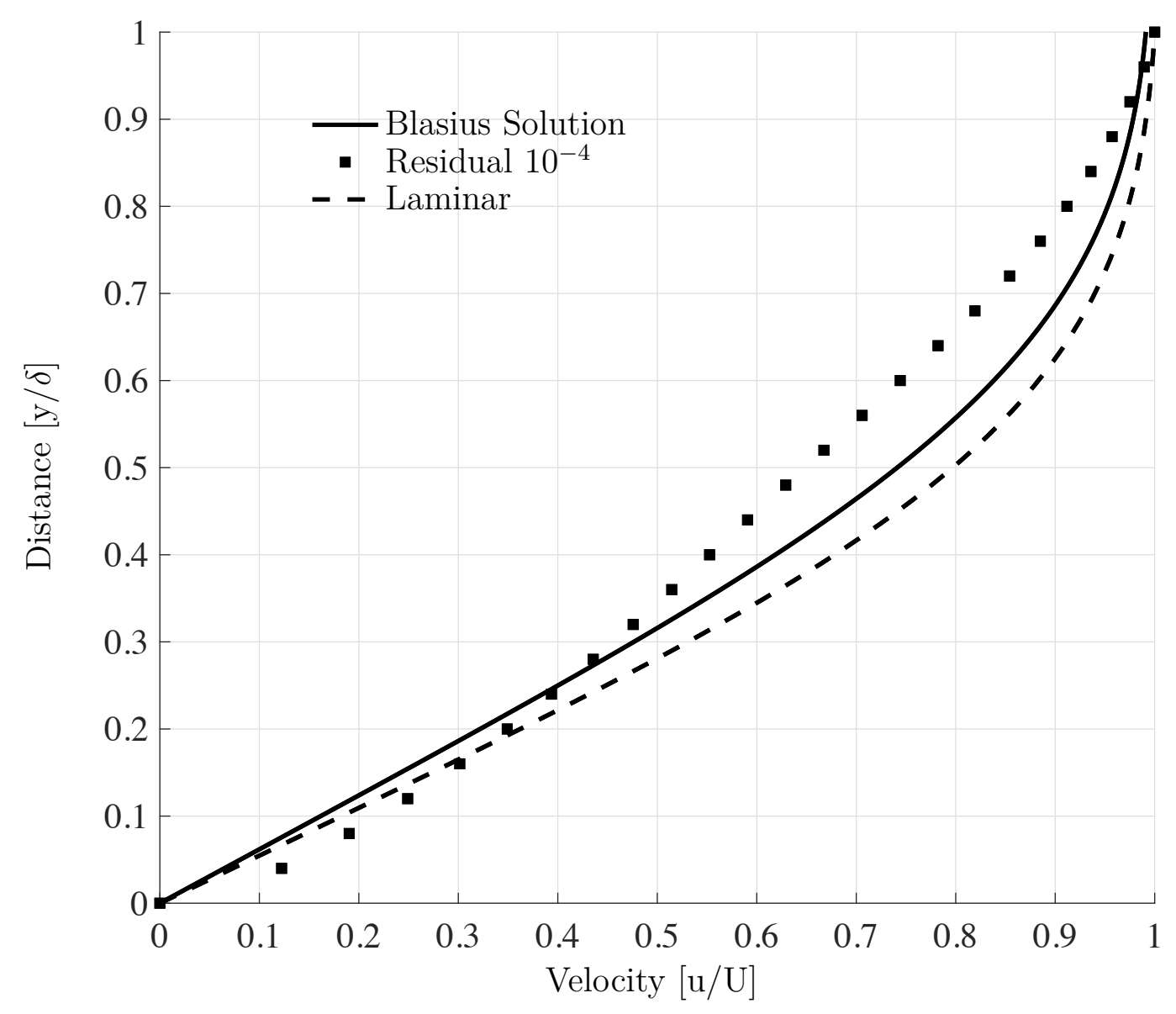

Figure 6: Boundary layer profiles of residual set to $10^{-4}$ and laminar condition compared to the Blasius solution.

$$
\delta^{*}=\int_{0}^{\infty}\left(1-\frac{u(y)}{U_{\infty}}\right) d y
$$

and the momentum thickness for incompressible flow is expressed as,

$$
\theta=\int_{0}^{\infty} \frac{u(y)}{U_{\infty}}\left(1-\frac{u(y)}{U_{\infty}}\right) d y
$$


A Blasius boundary layer has a shape factor of $H=2.59$. Calculating the displacement thickness and momentum thickness using Equation 54 and 55, the shape factor of each boundary layer profile is calculated. The shape factors for the residuals of $10^{-4}$ is calculated to be $H=2.26$, slightly lower than the Blasius solution indicating a more turbulent flow regime is present in the simulations. The fractional difference between this shape factor and the Blasius solution is $13 \%$. The difference observed with the comparison of the turbulent case and the Blasius solution show errors due to the presence of the turbulence model.

The simulation with the turbulence model turned off provides a shape factor of $H=2.51$, resulting in a difference of $3 \%$ in comparison to the Blasius solution. This case shows little variation when compared to the Blasius solution indicating that the equations used within SIMPLE solver are correct.

A case with the final residual set to $10^{-5}$ is tested and it is found to have only small differences between the two residual settings. Both profiles are found to have the same value of shape factor. This indicates that a final residual of $10^{-4}$ produces an acceptable result relative to that of the $10^{-5}$. The remaining simulations are completed with the lower residual setting of $10^{-4}$ to save computational time.

\subsubsection{Turbulent Boundary Layer Comparison}

In addition to the comparison to the Blasius solution the boundary layer profile is also compared to a turbulent boundary layer. No exact turbulent boundary layer 
profile exists, however several empirical models can approximate the boundary layer profile. The Van Driest method is a mean flow model for eddy viscosity and mixing length. This model is compared to a boundary layer taken from the simulation.

The turbulent boundary layer model consists of three sections. The shear layer, the overlap region and the outer region. Each of these sections is defined by its own equation expressed in Equations 56, 57 and 58. The shear layer is closes to the wall and has a linear relationship and extends from the surface to the non-dimensionalized value of distance value of $y^{+}=5$.

$$
u^{+}=y^{+}
$$

Where $u^{+}$is the quotient of the velocity $u$ and the shear velocity $u_{*}$. The overlap region has the equation developed by Van Driest [51]. The region continues upwards from $y^{+}=5$ to a level of $y^{+}=150$.

$$
\frac{d u^{+}}{d y^{+}}=\frac{2}{1+\sqrt{\left.1+4 \kappa^{2}\left(y^{+}\right)^{2}\left[1-\exp \left(-y^{+} / A^{+}\right)\right]^{2}\right)}}
$$

Equation 57 is solved to find the $u^{+}$value through the range of $y^{+}$. The constants within the equation are defined such that the equations match empirical data, are taken as: $\kappa=0.41$ and $A^{+}=24$. The outer region of the boundary layer is defined using a logarithmic equation where $y^{+}>150$. 


$$
u^{+}=\frac{1}{\kappa} \log \left(y^{+}\right)+C
$$

Similar to the $A^{+}$constant the value of $C$ is defined such that the equations match empirical data [51]. The complete range of $u^{+}$values now collected are converted to velocity and distance values for comparison to the simulated data. Both $u^{+}$and $y^{+}$ are functions of the friction velocity $u_{*}$. This value is calculated from the edge of the boundary layer where the value of velocity $u$ is equal to the free stream velocity $U$. Using the $u^{+}$value at the edge of the boundary layer, $u_{e}^{+}$, found from Equation 58 for $y^{+}=1900$,

$$
u_{*}=\frac{U}{u_{e}^{+}}
$$

With the friction velocity, the values of $u$ and $y$ are calculated for a flat plate parallel to the $70 \mathrm{~m} / \mathrm{s}$ flow $13.9 \mathrm{~cm}$ from the leading edge. This boundary layer profile is taken from a control case of the results presented in a later section. The values are then non-dimensionalized and compared to the simulation data in Figure 7.

Figure 7 shows that the Van Driest model predicts greater velocity in the near wall section, and less velocity in the mid-range of the boundary layer when compared to the simulation results. Similar to the Blasius solution the shape factor of the velocity profiles is compared between the Van Driest model and the simulation data. The Van Driest model proves to have a shape factor of $H=1.29$ and the simulation data $H_{13.9}=1.56$. The fractional difference in shape factor is found to be $22 \%$. 


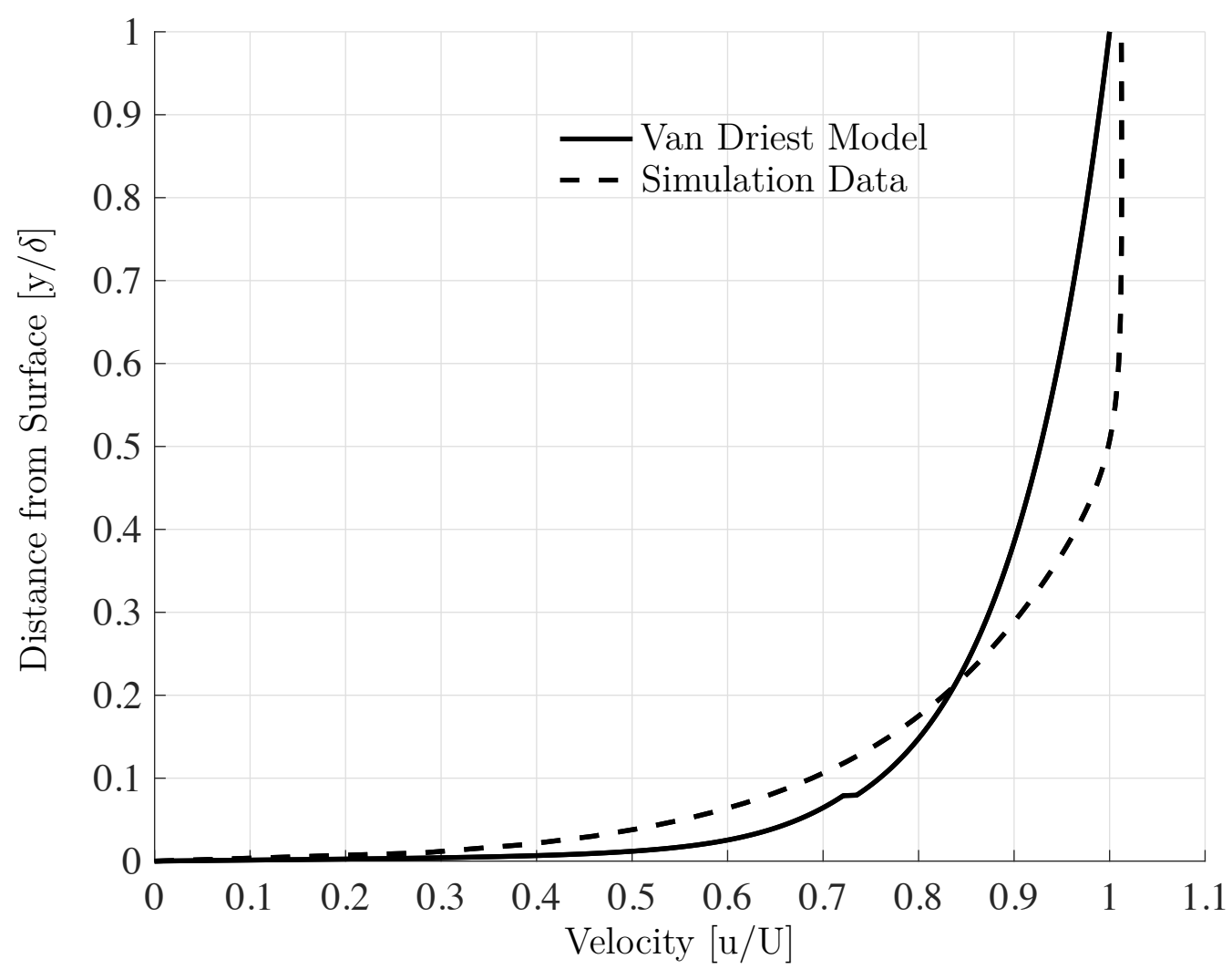

Figure 7: Van Driest turbulent boundary layer model and simulation data.

This difference is due to the fact that this boundary layer is not fully developed at this location and thus providing a higher shape factor value than that of the numerical solution. This is shown through the calculation of the shape factor at locations of $12 \mathrm{~cm}$ and $13 \mathrm{~cm}$ from the leading edge. Slightly up stream of the above shown boundary layer profiles. The location of $12 \mathrm{~cm}$ was found to produce a shape factor of $H_{12}=1.62$ while the $13 \mathrm{~cm}$ location provided a value of $H_{13}=1.61$. The decreasing trend across these three values indicates that the boundary layer is not 
fully developed.

\subsection{Validation}

The simplified plasma actuator boundary condition developed in this analysis is compared to experimental scenarios from the literature to ensure that similar flow structures are observed. The two dimensional domain is used to examine the effect of the plasma actuator on the surrounding air in quiescent conditions. This represents the rocket fin as a flat plate with a free stream velocity of zero. Past experiments examine the velocity profile produced by DBD plasma actuators in these conditions such as Akansu [21] and Jolibois [38] and many others.

The velocity profile from the quiescent simulation is measured to a height of 7 $\mathrm{mm}$ above the rocket fin at $7 \mathrm{~mm}$ from the plasma actuator boundary condition. This location is outside the boundary condition and therefore the velocity at the surface goes to the normal no slip condition at the surface. The resulting velocity profile is compared to experiments based on the magnitude of the induced velocity and the height from the surface of the fin at which the maximum induced velocity occurs. In Figure 8 the distance away from the fin is depicted in the $\mathrm{y}$ axis while the velocity induced by the plasma actuator is shown on the $\mathrm{x}$ axis.

The simulation case is run with the plasma actuator boundary condition velocity set at $-5 \mathrm{~m} / \mathrm{s}$ on the surface over a length of $5 \mathrm{~mm}$. The data collected from the quiescent

simulation are compared to the data collected by Akansu [21] using a pitot tube to 
measure dynamic pressure over the surface before converting it into velocity. The experimental data examines an excitation voltage of $7 \mathrm{kV}$ P-P at a frequency of $4 \mathrm{kHz}$.
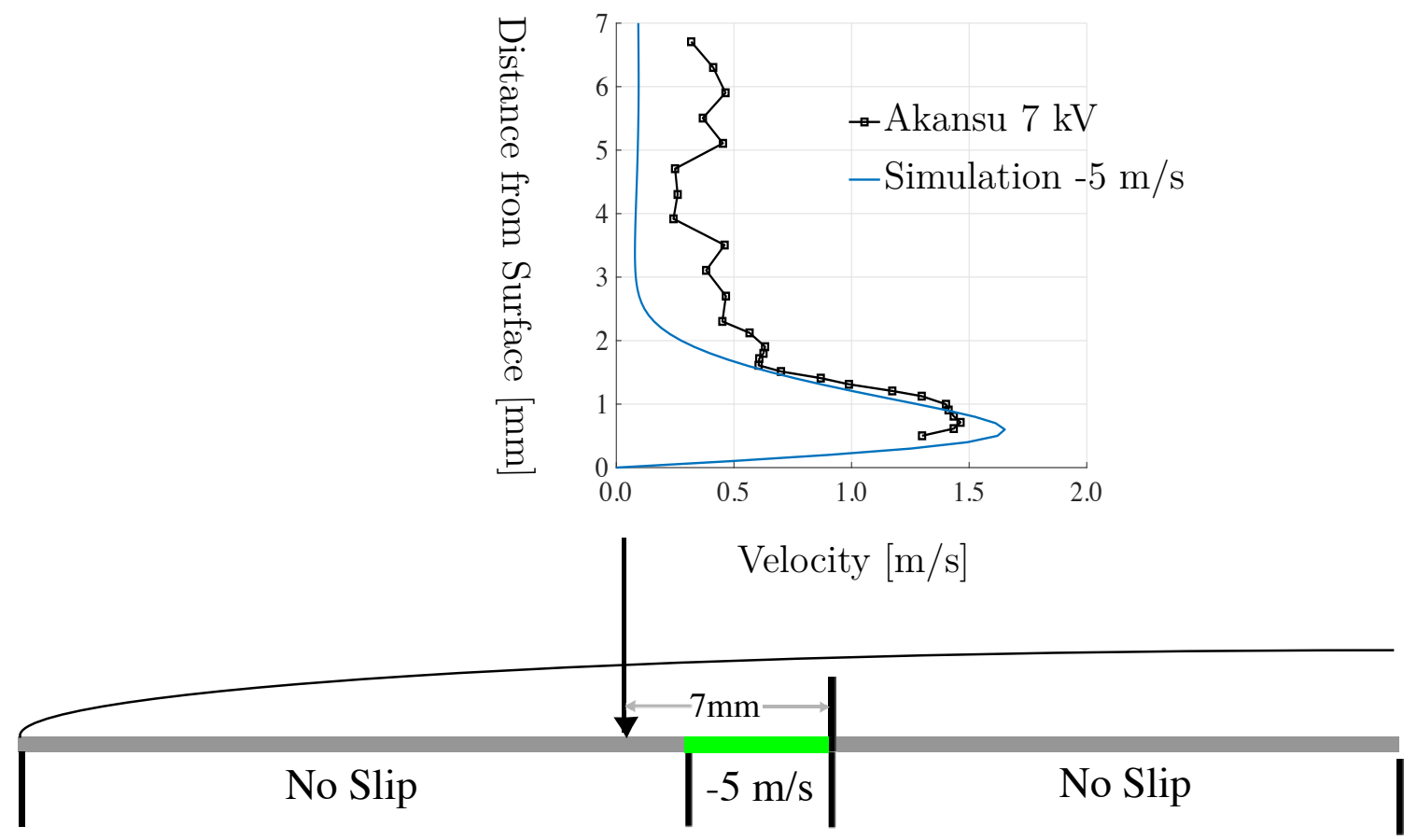

Figure 8: Quiescent OpenFOAM simulation compared to two plasma actuator experiments [21]

A secondary simulation is examined with the surface velocity of $-6 \mathrm{~m} / \mathrm{s}$. It is found that the boundary condition matches closely with Akansu's experiment with $8 \mathrm{kV}$ $\mathrm{P}-\mathrm{P}$ and $4 \mathrm{kHz}$ frequency, Figure 9 .

With the boundary condition set to $-6 \mathrm{~m} / \mathrm{s}$, the simulation shows that the induced velocity flow profile matches closely to the experimental velocity data provided by the experiments performed by Akansu for a voltage of $8 \mathrm{kV}$ P-P. The $-5 \mathrm{~m} / \mathrm{s}$ boundary condition slightly over-predicts the induced velocity profile of the $7 \mathrm{kV}$ 

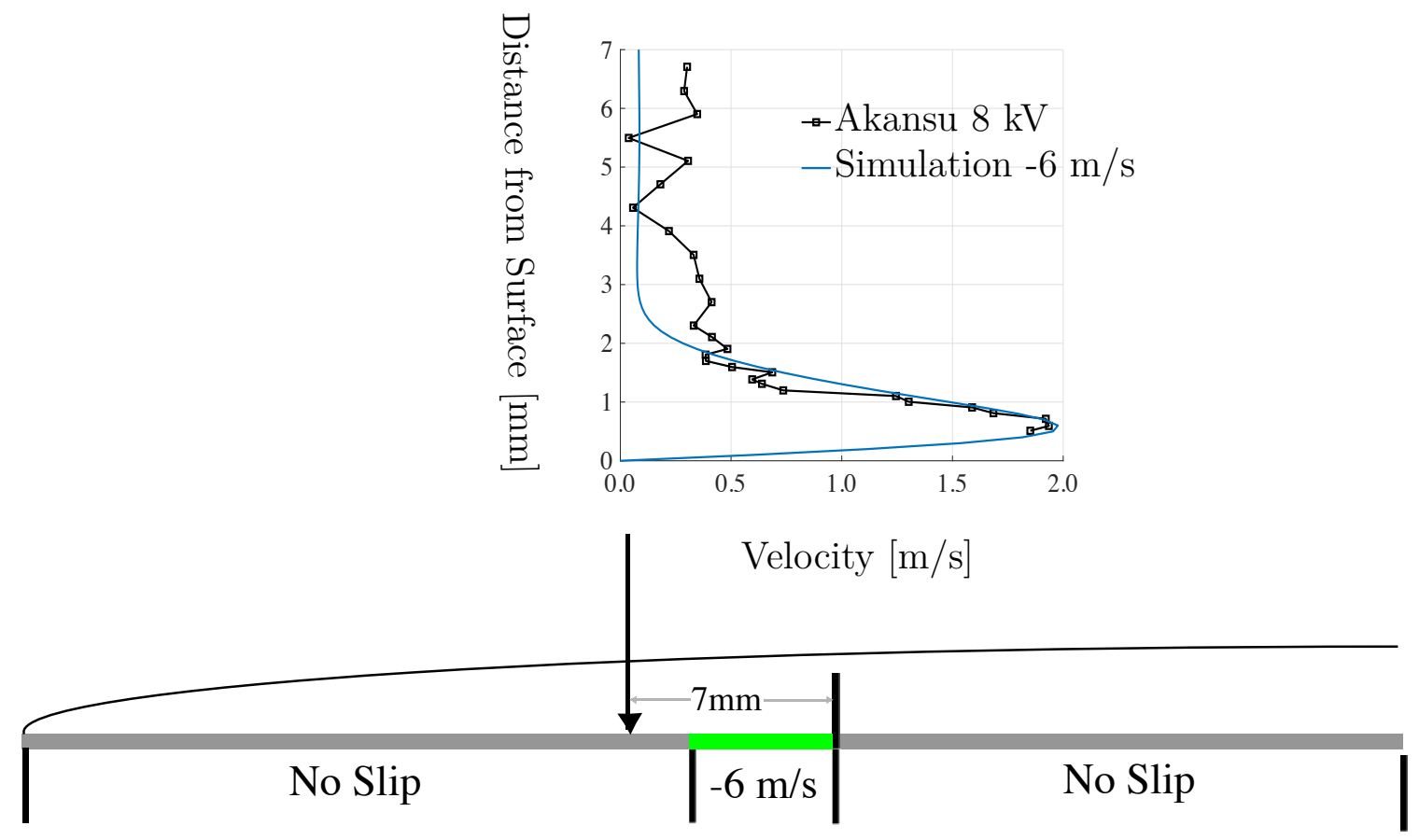

Figure 9: Quiescent OpenFOAM simulation compared to two plasma actuator experiments [21]

P-P experiment. Matching the velocity profiles to two different experiments with different boundary conditions not only indicates that this boundary condition is an acceptable representation of a DBD plasma actuator, but also that the boundary condition can be adjusted to match different parameters of plasma actuation. However, the robustness and flexibility of this boundary condition are not explored further here. 


\subsection{Mesh Convergence}

To ensure that the results gathered from the simulations are performed with an appropriately sized mesh, the number of cells within the mesh is varied. The number of cells within the grid is increased and decreased by a factor of two on the $\mathrm{X}$ and $\mathrm{Z}$ axis, resulting in a change in the number of cell on the order of four. The meshes examined are sized and labelled according to Table 2.

Table 2: Details pertaining to the course medium and fine mesh used in the grid convergence analysis

\begin{tabular}{|c||c|c|c|}
\hline Mesh Name & Coarse & Medium & Fine \\
\hline Mesh Size & 105,000 & 420,000 & $1,680,000$ \\
\hline Designation & 3 & 2 & 1 \\
\hline
\end{tabular}

The quiescent case is performed through the two new domains. The resulting boundary layer velocity profiles are examined at $7 \mathrm{~mm}$ from the simulated boundary condition shown in Figure 10. The boundary layer velocity profile for the medium mesh shown in this figure is the same as that shown in Figure 8.

Each of the three boundary layers examined with the three meshes is similar from

the surface stretching upwards to $2 \mathrm{~mm}$. A slight variation in maximum velocity is observed for each case. The greatest difference between the three cases ranges from $2 \mathrm{~mm}$ from the surface upwards. The course mesh is seen to show negative velocity while the medium mesh and the fine mesh maintain positive velocity through this range. 


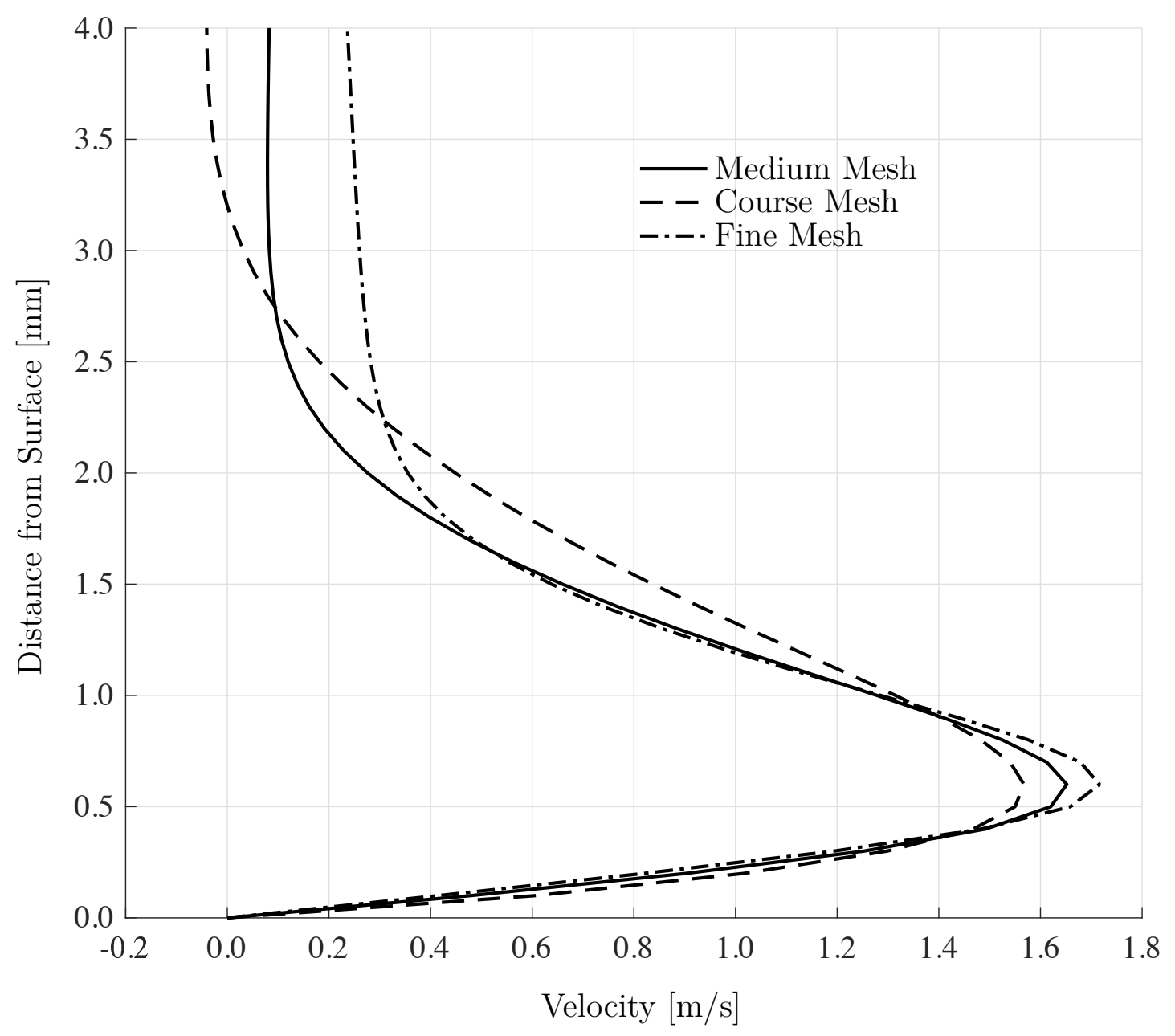

Figure 10: The boundary layers taken from the coarse medium and fine mesh simulations performed in quiescent air.

These meshes are further analyzed using a Richardson extrapolation to estimate the continuum value that would occur for a grid spacing of zero distance. The comparison of the grid uses a parameter defined as $f$ from the simulation results. In this case the maximum velocity of the induced flow measured at $7 \mathrm{~mm}$ downstream 
of the actuator boundary condition is used. The maximum induced velocity at this location for the course, medium and fine mesh are $f_{3}=1.57 \mathrm{~m} / \mathrm{s}, f_{2}=1.65 \mathrm{~m} / \mathrm{s}$ and $f_{1}=1.72 \mathrm{~m} / \mathrm{s}$ respectively. The velocity difference between the medium and fine mesh is approximately $0.06 \mathrm{~m} / \mathrm{s}$, a difference of $3.8 \%$. While the course mesh differs by approximately $0.08 \mathrm{~m} / \mathrm{s}$ indicating a difference of $5.4 \%$.

These values of maximum velocity can be applied to the Richardson extrapolation equation, Equation 60 states that the value for a continuous grid $f_{\infty}$ is a function of the fine mesh $f_{1}$ the regular mesh $f_{2}$ and the ratio between the grid sizes $r$.

$$
f_{\infty}=f_{1}+\frac{f_{1}-f_{2}}{r^{p}-1}
$$

The value of $p$ is the order of convergence calculated as,

$$
p=\ln \left(\frac{f_{3}-f_{2}}{f_{2}-f_{1}}\right) \frac{1}{\ln (r)}
$$

Due to the doubling of the mesh size in both directions the grid size fraction is $r=2$. Using Equation 60 the continuous maximum velocity, $f_{\infty}$ is found to be 1.92 $\mathrm{m} / \mathrm{s}$. The same result is found when using the peak velocity differential from the medium and the course meshes. Therefore, using a medium grid suggests a grid induced error of approximately $14 \%$.

With the input of maximum induced velocity and the grid refinement ratio Equation 
61 produces $p=0.39$. The order of convergence is used to calculate the grid convergence index (GCI) in Equation 62

$$
G C I=\frac{F_{a}|\epsilon|}{r^{p}-1}
$$

In which $F_{a}$ is the safety factor set to equal 1.25 for comparisons for three grids or more, and $\epsilon$ is the relative error of $14 \%$, calculated above. For the course mesh comparison to medium mesh it is found $G C I_{23}=0.22$. For the medium mesh to that of the fine mesh $G C I_{12}=0.15$ is found.

A simple check is performed to examine if the grid convergence study produces values that are within the asymptotic range of convergence, Equation 63. The GCI values calculated above will produce a value near 1.0 if the asymptotic range is met.

$$
e r r=\frac{G C I_{23}}{r^{p} G C I_{12}}
$$

Inputting the GCI values found for this grid convergence study it is found that err $=1.09$, which is approximately equal to 1 indicating that the medium grid is within the asymptotic range. It is therefore concluded that medium grid is of sufficient size to collect acceptable results. With this study the results presented can expect an error of $14 \%$. 


\section{Chapter 4}

\section{Results}

The purpose of the CFD simulations is to estimate the feasibility of using plasma actuators to induce roll on a sounding rocket. Future plans to field test the plasma actuators on a sounding rocket are currently being developed in partnership with UNB. Before the field test a predictive basis to how much roll can be induced

must be developed. These CFD simulations will provide a basis of knowledge of what can be expected during the sounding rocket launch. Two stages of CFD simulations are used to examine multiple variables within the experiment. The first stage examines the pressure distribution and the resulting force on the fin from the activation of the plasma actuator with respect to its location on the surface of the fin. The second stage examines the resulting force on the fin at the as-built plasma actuator location with respect to a range of velocities within the two dimensional domain. These results are paired with the predicted trajectory of the sounding rocket to estimate the induced roll rate due to plasma actuation. 


\subsection{Actuator Location}

The simulations regarding the resulting force with respect to location are conducted with a free stream velocities of $20 \mathrm{~m} / \mathrm{s}$ and $70 \mathrm{~m} / \mathrm{s}$. The boundary condition representing the plasma actuator is set as the surface of the fin having a tangential velocity of $5 \mathrm{~m} / \mathrm{s}$ in the upstream direction, as done in the previous section. This boundary condition is set at 14 locations on the surface ranging from $25 \%$ chord to $90 \%$ chord in $5 \%$ chord increments. The resulting forces are compared to find the location that produces the highest forces.

To compare the effect of the plasma actuator location on the force applied to the fin each actuator location is examined within the same bounds. The boundaries in which the pressure distribution is examined is between $13.5 \mathrm{~mm}$ upstream and 4 $\mathrm{mm}$ downstream of the trailing edge of the actuator. This range is established by observing where the pressure differential between an actuated and an un-actuated simulation shows a variation greater than $1 \mathrm{~Pa}$. This value is used to reduce any numerical errors that may occur within the simulations.

Integrating the pressure with respect to chord-wise distance results in a force per span-wise distance value using the equation,

$$
F_{m}=\int_{x-4 m m}^{x+13.5 m m}\left(P_{\text {noact }}-P_{\text {act }}\right) d x
$$

These force values are compared across all examined actuator locations. The 
value of $P_{\text {noact }}$ is determined from simulating an identical domain and geometry but with a no-slip condition applied along the entire fin, (the surface velocity set along the length of the boundary condition is removed and set to a no slip condition).

Each of the examined actuator locations has a similar pressure distribution. Figure 11 displays the pressure distributions over each examined location within the bounds of the integration described previously. It is observed that approaching the leading edge of the actuator there is an increase in pressure relative to the non-actuated case. Above and trailing the actuator there is a larger low pressure region. The result is a lower overall pressure on the surface to which the plasma actuator is mounted relative to the un-actuated surface.

The pressure distribution is further examined in the form of a pressure contour plot. Figure 12 shows the pressure distribution over the simulated plasma actuator with the flow moving from left to right for the case of $20 \mathrm{~m} / \mathrm{s}$ and the actuator located at $50 \%$ chord. The figure shows the small high pressure zone approaching the leading edge of the actuator and the relatively larger low pressure zone over top of and trailing the actuator.

The lower velocity flow in the boundary layer meets the opposing induced velocity of the plasma actuator resulting in a region of higher pressure. A portion of the lower boundary layer creates a vortex over the simulated actuator while another portion is directed upward over the actuator. This upward flow moves over the vortex creating the low pressure zone and the resulting lift. 

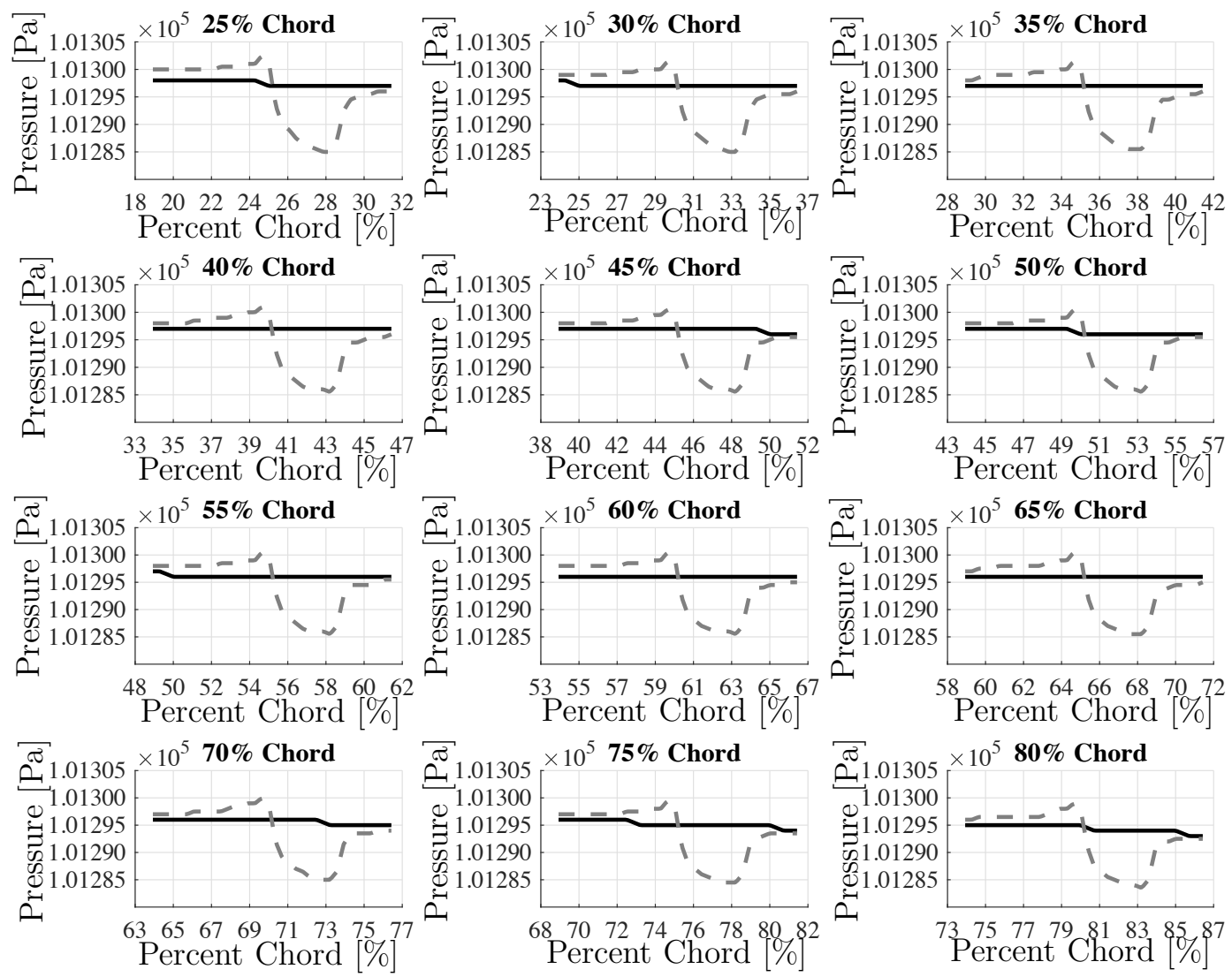

Percent Chord [\%]
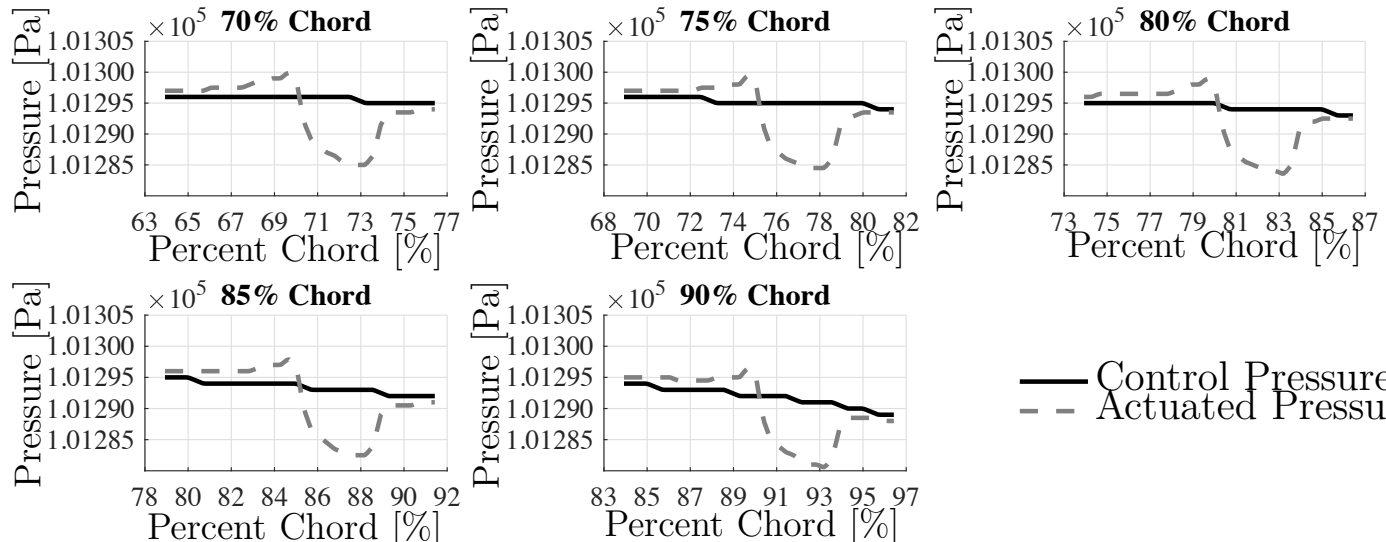

Percent Chord $\%$ ]

Percent Chord [\%]

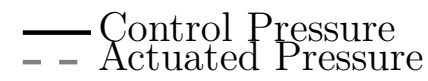

Figure 11: Pressure distributions over the simulated actuator and control for each examined chord-wise location located, at free stream velocity of $20 \mathrm{~m} / \mathrm{s}$. 


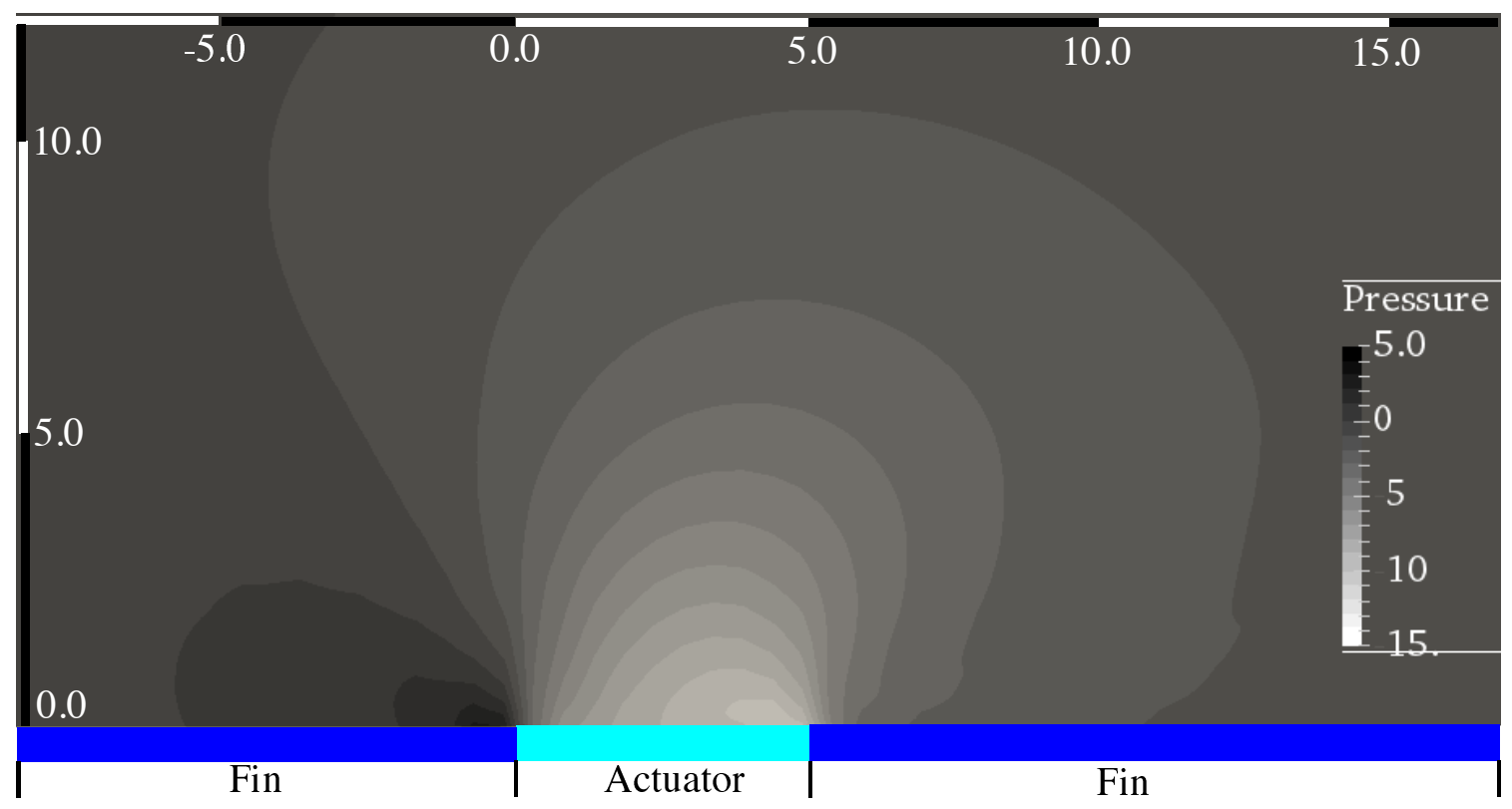

Figure 12: Relative pressure over the plasma actuator in Pascals, distances for scale in millimetres, free stream velocity of $20 \mathrm{~m} / \mathrm{s}$.

The resulting pressure differential between the actuated and un-actuated surfaces of the rocket fin are shown in Figure 13. These force values are found at a free stream velocity of $20 \mathrm{~m} / \mathrm{s}$, with the plasma actuator at locations varying between $25 \%$ to $90 \%$ of the chord.

Figure 13 shows that the resulting force acting on the fin is dependent on its chord-wise location. The highest induced force occurs at $40 \%$ chord to $45 \%$ chord. The same range of actuator locations are examined at a free stream velocity of 70 $\mathrm{m} / \mathrm{s}$. It can be observed that the best location is between $35 \%$ and $45 \%$ chord sown 


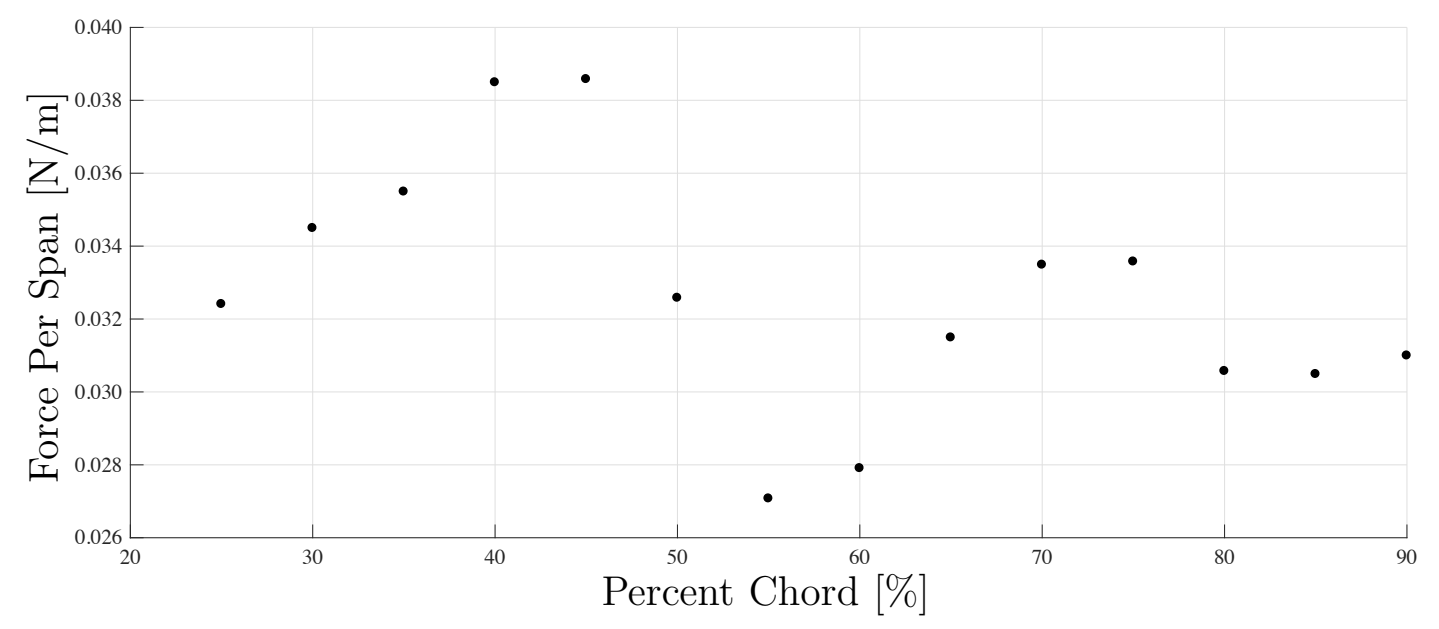

Figure 13: Force per span-wise distance as a function of chord-wise location of the actuator at $U_{\infty}=20 \mathrm{~m} / \mathrm{s}$.

in Figure 14. This indicates that the best location of the plasma actuator is only weakly dependent on the free stream velocity.

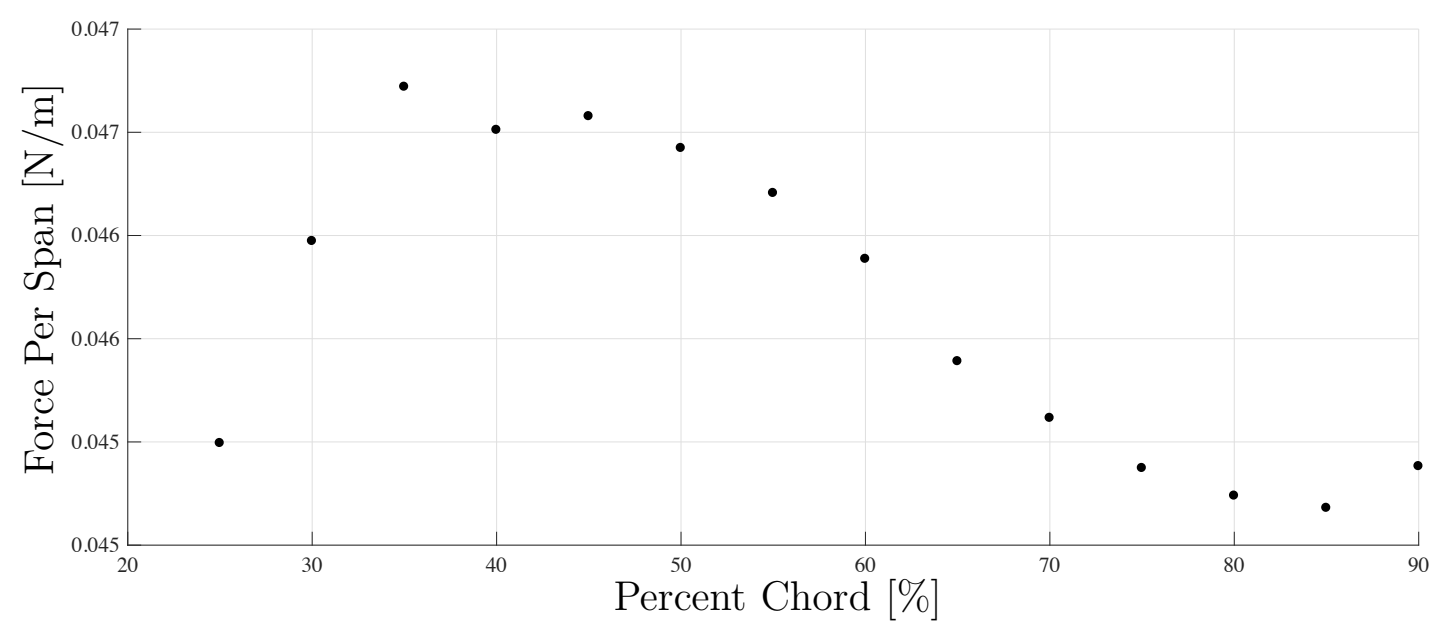

Figure 14: Force per span-wise distance as a function of chord-wise location of the actuator at $U_{\infty}=70 \mathrm{~m} / \mathrm{s}$. 
The $50 \%$ location is used in the following simulations based on an as-built fin constructed for laboratory testing before the field test is executed with the plasma actuators on the sounding rocket. This location is not ideal in that it does not provide the highest force per span-wise distance values as shown by the two free stream velocities tested. Placement to achieve the highest force per span-wise meter values is shown to be the $40 \%$ to $45 \%$ chord location. However, the plasma actuator in this location does produce high values of force relative to other examined locations particularly at higher velocity.

\subsection{Normal Force with Respect to Velocity}

The force applied to the fin by an actuator at $50 \%$ chord is determined through a range of velocities, between $10 \mathrm{~m} / \mathrm{s}$ and $90 \mathrm{~m} / \mathrm{s}$ in $10 \mathrm{~m} / \mathrm{s}$ intervals. These results are used to evaluate the effect of the plasma actuator with respect to free stream velocity. This velocity range represents a significant portion of the sounding rocket trajectory while remaining near the velocities examined in the literature and, allowing for the use of incompressible CFD solvers by remaining below 0.3 Mach. The $50 \%$ chord location of the actuator is used in the same computational domain as used to evaluate the force characteristic to actuator location. The force for each velocity is found using the same integration process shown in Equation 64. The resulting force with respect to the velocity curve from these simulations is used to calculate the induced roll rate of the rocket. 
The sounding rocket will coast from the engine burnout to apogee, during which the plasma actuators mounted in the fins will be activated. Simulating the effect of the plasma actuator through the range of velocities experienced by the rocket allows for the development of a force with respect to velocity curve. These results are used with the estimated trajectory found in the following section to provide the predictive basis for the roll rate that will be observed in the field test.

The force with respect to velocity curve created from simulations is shown in Figure 15. As the rocket decelerates the plasma induced force per span-wise distance acting on the fins decreases. A polynomial trend line through the data points allows for the estimated force to be calculated with respect to velocity curve. The trend line fits with an R-squared value of 0.99 indicating a close fit to the data set.

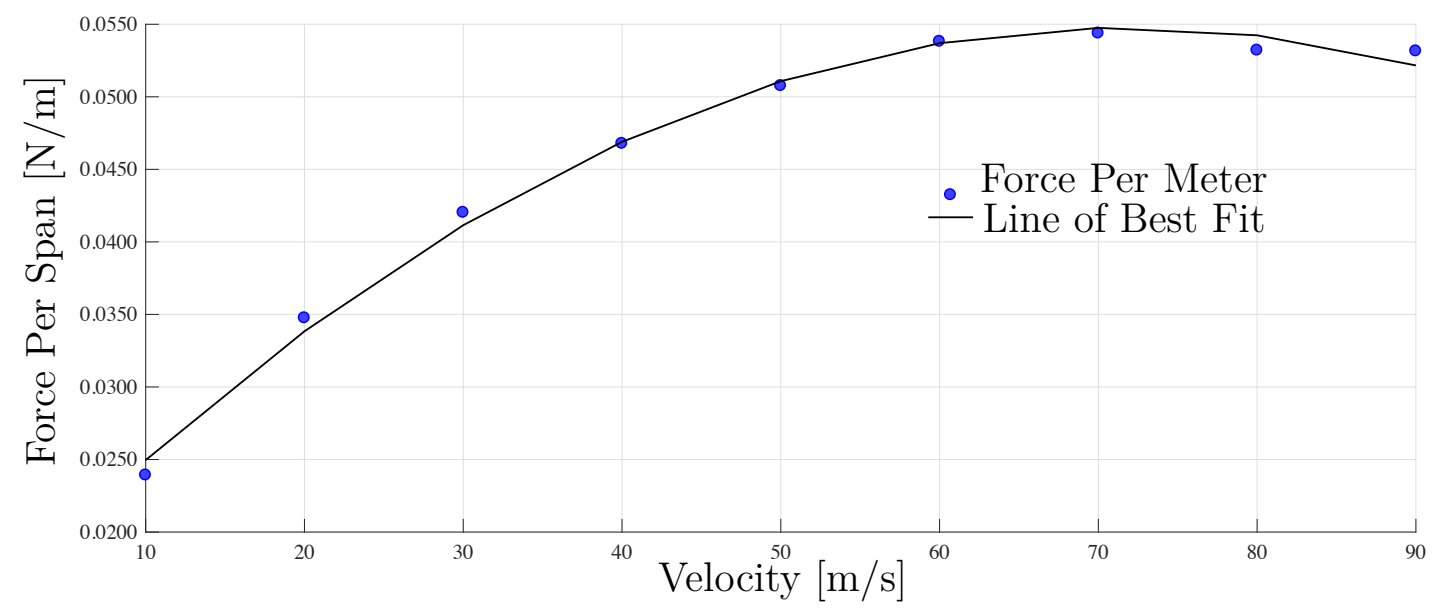

Figure 15: The force acting on the fin for velocities ranging $10 \leq U_{\infty} \leq 90$.

The equation of the trend line is expressed as the force per span-wise distance $F_{m}$ 
with respect to the velocity $U(t)$, expressed in Equation 65

$$
F_{m}=-7.82 \times 10^{-6} U(t)^{2}+1.1 \times 10^{-3} U(t)+1.45 \times 10^{-2}
$$

The sounding rocket is designed to have a plasma actuator mounted to the fins spanning a distance of $b_{a}=0.144 \mathrm{~m}$ across the surface, Figure 4 . Vorobiev et al. [43] found that plasma actuators produce a constant lift increase across their span when placed on airfoils. Therefore, it is assumed that the force per span-wise meter across the fin is constant. Equation 65 is therefore multiplied by the span of the actuator to calculate the force $F$ acting on the fin with respect to velocity resulting in the equation,

$$
F=-1.12 \times 10^{-6} U(t)^{2}+1.61 \times 10^{-4} U(t)+2.09 \times 10^{-3}
$$

This resultant force is assumed to act at the centre of the fin's span. The total span of the fin is $b_{f}=0.168 \mathrm{~m}$, as observed in Figure 16, the mid-span length is then $b_{f} / 2=0.084 \mathrm{~m}$. The torque arm of the resulting plasma actuated force is then the sum of the mid-span length and the radius of the rocket fuselage, $r=0.076 \mathrm{~m}$ resulting in a total torque arm length $L=0.16 \mathrm{~m}$.

The torque of one fin, $\tau_{f}$, with respect to velocity equation is found by multiplying the torque arm length by force with respect to velocity formula, or $\tau_{f}=F \times L$, which produces 


$$
\tau_{f}=-1.80 \times 10^{-6} U(t)^{2}+2.58 \times 10^{-5} U(t)+3.34 \times 10^{-4}
$$

The sounding rocket is designed to have three fins. To induce roll symmetrically a plasma actuator is mounted on all three of the rocket's fins. The total torque, $\tau$, acting on the rocket is therefore the sum of the three torques, $\tau=3 \tau_{f}$ creating the equation,

$$
\tau=-5.40 \times 10^{-7} U(t)^{2}+7.75 \times 10^{-5} U(t)+1.0 \times 10^{-3}
$$

The torque is related to the angular acceleration, $\ddot{\phi}$, according to the formula,

$$
\tau=I \ddot{\phi}
$$

Where I is the moment of inertia of the object. Solving this equation for the angular acceleration yields

$$
\ddot{\phi}=\frac{\tau}{I}
$$

The moment of inertia of the rocket is calculated with the assumption that the rocket can be depicted as a thin cylinder of mass $m=13 \mathrm{~kg}$ and radius $r=0.076 \mathrm{~m}$. The resulting moment of inertia of the sounding rocket is calculated to be 


$$
I=m r^{2}=0.075 \mathrm{kgm}^{2}
$$

Substituting the results of Equation 71 into Equation 70 yields the angular acceleration with respect to velocity formula,

$$
\ddot{\phi}=-7.21 \times 10^{-6} U(t)^{2}+1.0 \times 10^{-3} U(t)+1.34 \times 10^{-2}
$$

The angular velocity of the rocket during the flight can be predicted using the velocity profile of the sounding rocket. Typical flight characteristics at burnout of velocity and altitude are provided by UNB yield an estimate of the trajectory and its velocity profile. Pairing the angular acceleration with respect to velocity with the velocity profile of the sounding rocket provides a basis to calculate the induced angular velocity of the rocket due to plasma actuation throughout the flight.

\subsection{Predicting Induced Angular Velocity}

The trajectory of the rocket is found through examination of the aerodynamic forces on the rocket. The rocket path is modelled using the initial conditions after the solid rocket engine burns out. The shape and mass of the vehicle are used to calculate the aerodynamic and gravitational forces applied to the rocket. UNB provides the engine burnout velocity of $220 \mathrm{~m} / \mathrm{s}$, and the burnout altitude of $440 \mathrm{~m}$ (this data 
having been collected from previous experimental flight tests). The aerodynamic drag forces are used to predict the velocity and altitude of the rocket during the coast from engine burnout to apogee.

The time between engine burnout and apogee, or the coast period, is found through combining the aerodynamic drag forces acting on the rocket and gravitational acceleration. The forces applied by the actuators are several orders of magnitude less than the aerodynamic forces and are therefore these forces are ignored for the purposes of the trajectory analysis. The velocity and altitude of the rocket can then be represented as a function of time to satisfy the angular acceleration formula given by Equation 72 .

At the moment of engine burn out the flight path is assumed to have an angle of $\theta=1 \times 10^{-4 \circ}$ from vertical at burnout such that both vertical and lateral velocities are calculated. The provided altitude and velocity values at engine burnout along with the flight path angle make up the initial conditions of the trajectory.

Due to the relatively low expected apogee of the rocket the air density and viscosity are assumed constant with altitude, and set to $\rho=1.225 \mathrm{~kg} / \mathrm{m}^{3}$ and $\mu=1.8428 \times 10^{-5} \mathrm{Ns} / \mathrm{m}^{2}$. Corresponding to the density and viscosity of dry air $15^{\circ}$ Celsius in standard sea level pressure. The drag forces acting on the rocket are assumed to be: shape drag for the frontal area of the fins and nose cone, and skin friction on the rocket fuselage. 
Accelerations are evaluated at each time step of length $1 \times 10^{-2}$ seconds from engine burn out to apogee. A shorter time step of $1 \times 10^{-3}$ seconds is found to result in a change in the estimated rotational velocity on the order of $1 \times 10^{-5} \mathrm{rad} / \mathrm{s}$, which is small relative to the final value. Therefore, the larger time step is chosen to reduce total file size.

The drag coefficient of the frontal area of the rocket nose cone is defined as $C_{\text {Dcone }}=$ 0.3 for a three dimensional cone with a half angle of $5^{\circ}$ with a frontal area of $S_{c}=$ $1.82 \times 10^{-2} \mathrm{~m}^{2}$ [53]. The coefficient of drag used for the fins is $C_{D \text { fin }}=0.75$ where the fin is assumed to be a wedge with a half angle of $7^{\circ}$, and a frontal area of $S_{f}=2.4 \times 10^{-3} \mathrm{~m}^{2}$. The dimensions of the rocket as provided by UNB are shown in Figure 16.

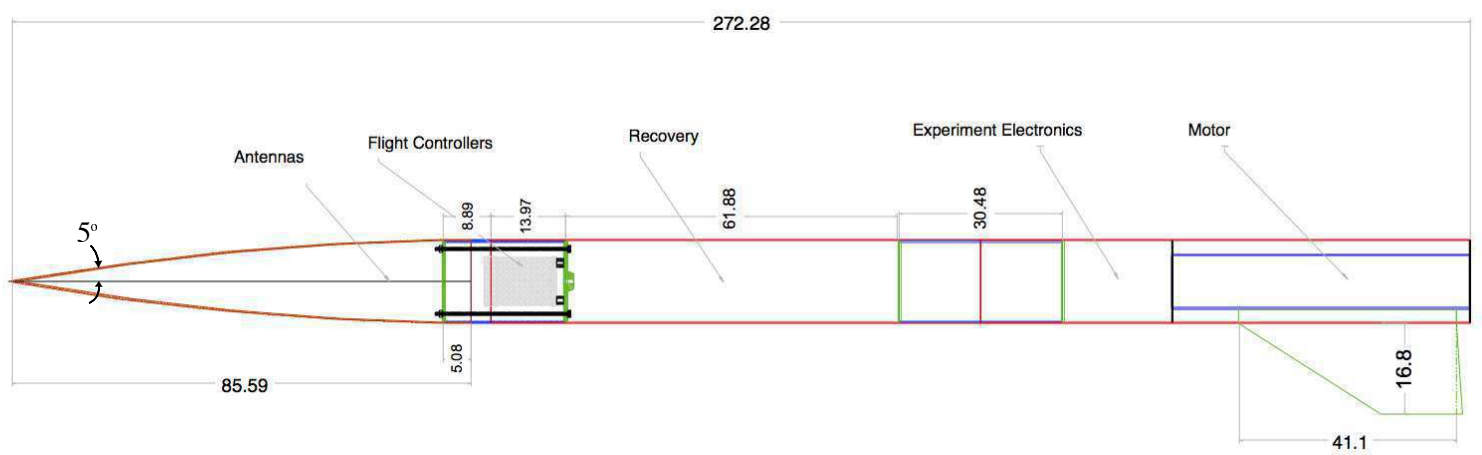

Figure 16: The sounding rocket's dimensions as provided by UNB [cm].

The force experienced due to shape drag, expressed below, is used to analyze the aerodynamic forces on the nose cone and the fins. 


$$
\begin{gathered}
F_{\text {dcone }}=\frac{1}{2} \rho C_{\text {Dcone }} U(t)^{2} S_{c} \\
F_{d f i n}=\frac{1}{2} \rho C_{D f i n} U(t)^{2} S_{f}
\end{gathered}
$$

The skin friction drag for the fuselage, $F_{\text {sfuselage }}$, is calculated using the coefficient of skin friction drag, $C_{s d}$, and the surface of the rocket fuselage. The fuselage has an area of approximately $S_{r}=0.8925 \mathrm{~m}^{2}$. The skin friction drag is therefore expressed as

$$
F_{s}=\frac{1}{2} \rho C_{s d} U(t)^{2} S_{r}
$$

where the coefficient of skin friction drag, $C_{s d}$, is found using the Prandtl Schlichting interpolation formula [54], given by

$$
C_{s d}=\frac{0.455}{\log \left(R_{e}\right)^{2.58}}
$$

The Reynolds number, $R_{e}$ is calculated separately according to the total length of the fuselage, $1.86 \mathrm{~m}$, and the current free stream velocity.

The sum of the aerodynamic forces on the rocket is found through the addition of all the calculated forces from Equations 73, 74 and 75.

$$
F_{\text {total }}=F_{s}+F_{d c o n e}+F_{d f i n}
$$


Which is used to determine the acceleration in $\mathrm{X}$ and $\mathrm{Z}$,

$$
\begin{gathered}
\dot{u}=\frac{F_{t o t a l} \sin \theta}{m} \\
\dot{w}=\frac{F_{g}+F_{\text {total }} \cos \theta}{m}
\end{gathered}
$$

The acceleration due to aerodynamic forces can be added to that of gravity, expressed in Equations 78 and 79, to find the accelerations along the $\mathrm{X}$ and $\mathrm{Z}$ axis according to Figure 17.

The acceleration in the $\mathrm{Z}$ direction found in Equations 79 is used to calculate the velocity, Equation 80, and position, Equation 81, at the current time step, $n$ from the information of the past time step $n-1$.

$$
\begin{aligned}
& w^{n}=w^{n-1}+\dot{w}^{n-1} \Delta t \\
& Z^{n}=Z^{n-1}+w^{n-1} \Delta t
\end{aligned}
$$

Similar equations are used on the $\mathrm{X}$ direction using the acceleration found from Equation 78 representing the horizontal component of the rocket trajectory,

$$
\begin{gathered}
u^{n}=u^{n-1}+\dot{u}^{n-1} \Delta t \\
X^{n}=X^{n-1}+u^{n-1} \Delta t
\end{gathered}
$$




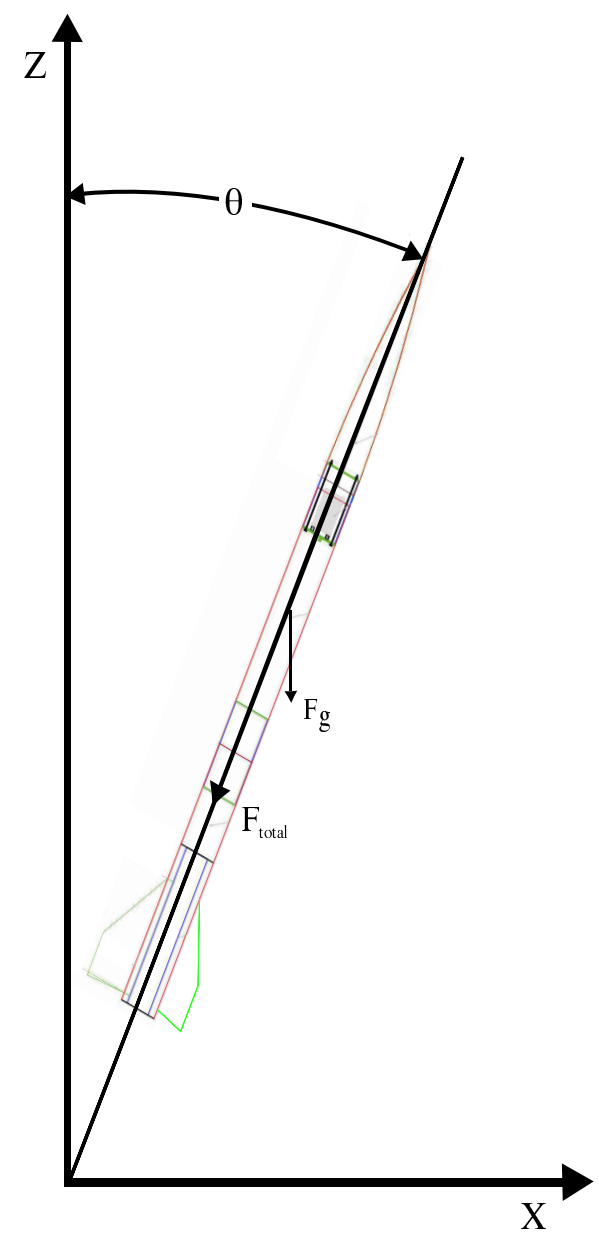

Figure 17: Acceleration and velocity in their component parts with flight path angle $\theta$

The new flight path angle is calculated using the arctangent of the newly calculated velocities,

$$
\theta^{n}=\tan ^{-1}\left(\frac{u^{n}}{w^{n}}\right)
$$

Using the calculated velocities at the time step $\mathrm{n}$, the forces are recalculated. This 
process creates the velocity with respect to time function of the rocket from burnout to apogee. The velocity calculated within the range of $90 \mathrm{~m} / \mathrm{s}$ to $10 \mathrm{~m} / \mathrm{s}$ examined by the CFD simulations is used to find the angular velocity induced by the plasma actuators.

The trajectory calculation estimates that the coast from engine burn out to apogee has a flight time of 14.6 seconds reaching an altitude of $1281 \mathrm{~m}$ above the engine burnout, $1721 \mathrm{~m}$ from launch. The predicted velocity of the vehicle is shown in Figure 18. The rocket slows to $U=90 \mathrm{~m} / \mathrm{s}$ at approximately 6.4 seconds after engine burnout and $U=10 \mathrm{~m} / \mathrm{s}$ after 13.6 seconds from burnout. Where,

$$
U=\sqrt{u^{2}+w^{2}}
$$

Between $90 \mathrm{~m} / \mathrm{s}$ and $10 \mathrm{~m} / \mathrm{s}$ there is an estimated total of 7.2 seconds of flight time during which the actuators are to be active and applying torque to roll the rocket. In the upper right corner of Figure 18 shows a magnified section of the velocity with respect to time curve is shown. Where the actuators are actively rolling the rocket.

The viscosity and density used in the trajectory analysis are assumed to be constant with altitude, producing the altitude as a function of time profile shown in Figure 19. An analysis using typical atmospheric densities shows the effect of changing air density on the trajectory of the rocket (a change in density from the assumed sea level density of $\rho=1.205 \mathrm{~kg} / \mathrm{m}^{3}$ to a density that can be expected at 1000 meters above sea level, $\rho=1.112 \mathrm{~kg} / \mathrm{m}^{3}$ and a corresponding change in viscosity from 


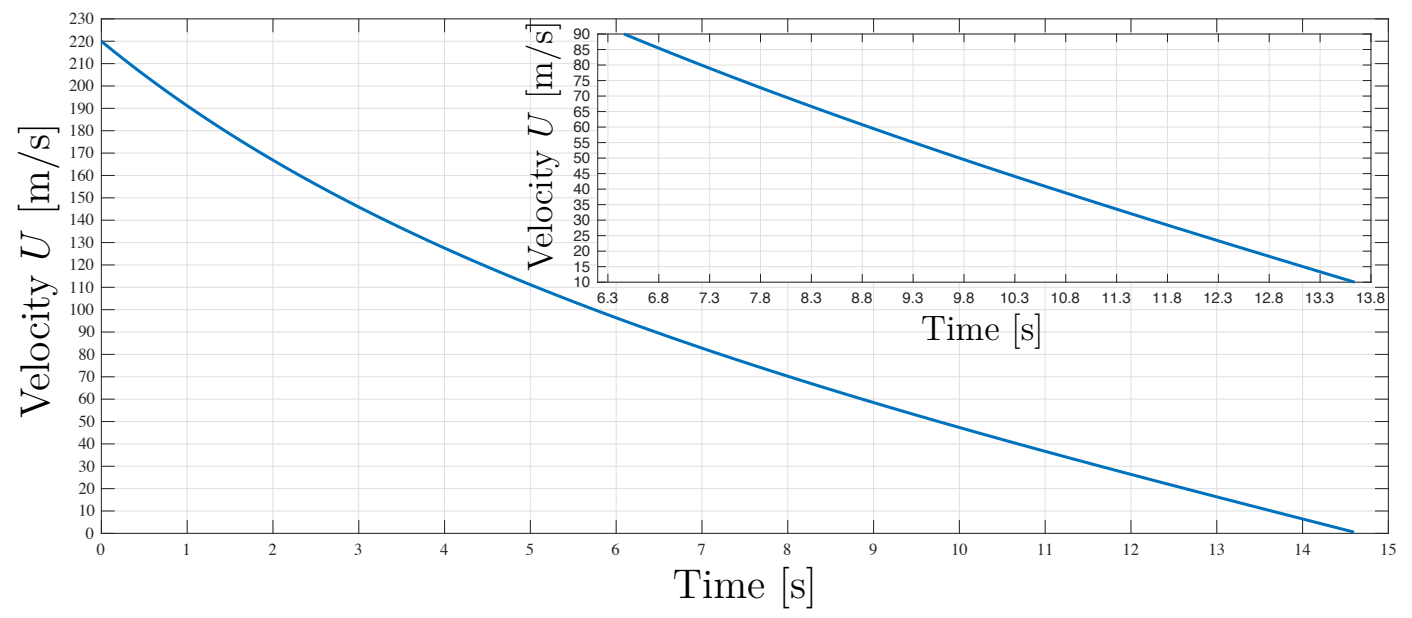

Figure 18: Predicted velocity of the rocket with a magnified sector where the plasma actuators are active.

$\mu=1.842 \times 10^{-5} \mathrm{Ns} / \mathrm{m}^{2}$ to $\left.\mu=1.758 \times 10^{-5} \mathrm{Ns} / \mathrm{m}^{2}\right)$ results in a change in apogee height of $50 \mathrm{~m}$. This value is only $2.7 \%$ of the total altitude and is considered to have an insignificant effect on the CFD simulations, thereby justifying the assumption of constant density with altitude. Furthermore, the values of $\rho=1.205$ and $\mu=1.842 \times 10^{-5}$ are the same as those used within the CFD simulations and are kept the same for the purpose of consistency.

The velocity data from this trajectory analysis is used to provide the velocity with respect to time curve, $U(t)$ in Figure 18. The resulting curve is paired with the angular acceleration as a function of velocity curve found in Equation 72. Both curves are then used to calculate the angular acceleration of the sounding rocket with respect to time. Integrating the angular acceleration provides the angular velocity of the rocket from burnout to apogee performed in Equation 86. 


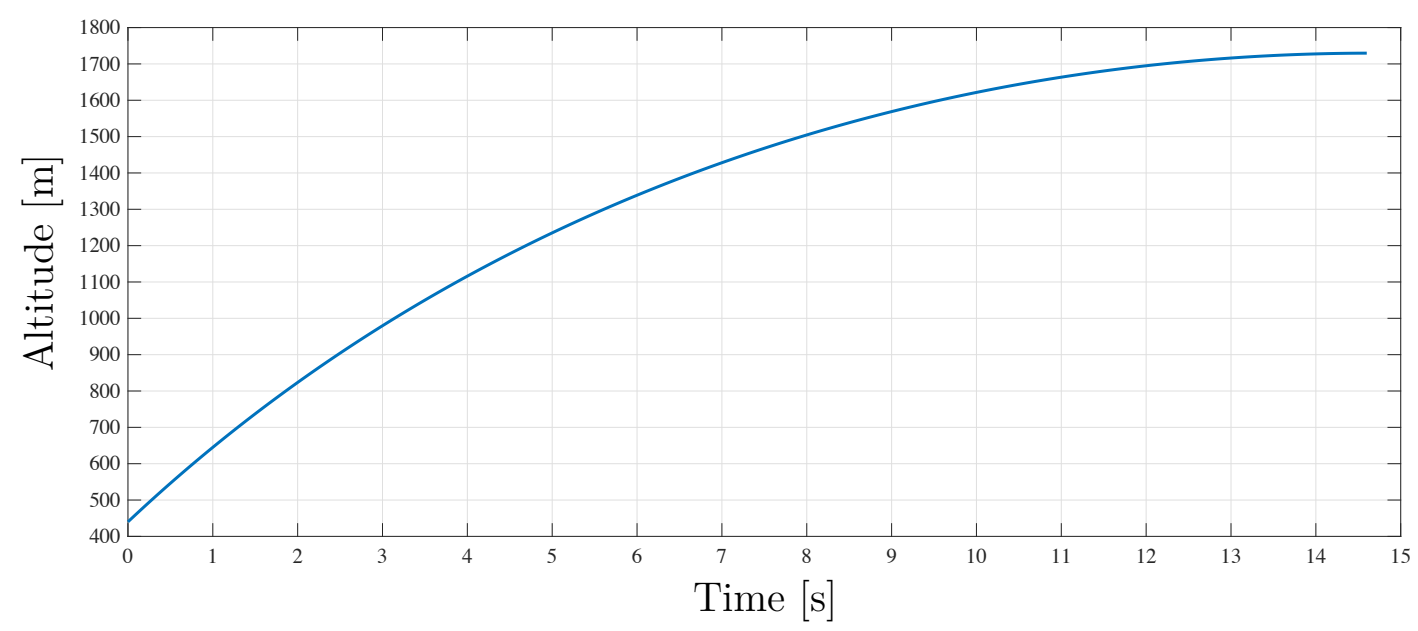

Figure 19: Predicted trajectory of the rocket.

$$
\dot{\phi}=\int_{\text {burnout }}^{\text {apogee }} \ddot{\phi} d t
$$

The resulting angular velocity increases almost linearly as shown in Figure 20. The maximum roll of the sounding rocket is found to be approximately $0.30 \mathrm{rad} / \mathrm{s}$ or $2.86 \mathrm{RPM}$ as the rocket reaches a velocity of $10 \mathrm{~m} / \mathrm{s}$.

The sounding rocket is planned to be fitted with an ADIS 16365 inertial measurement unit (IMU) with a resolution of $0.0125 \%$ s, an expected noise of $0.8 \% \mathrm{~s}$ rms and a non-linearity of $0.1 \%$ of full scale. This results in a total uncertainty of $0.804 \%$ or $0.134 \mathrm{RPM}$. The ADIS $16365 \mathrm{IMU}$ is therefore sensitive enough to distinguish the signal from the noise. This IMU can be used to validate the predictions produced through the CFD simulations. 


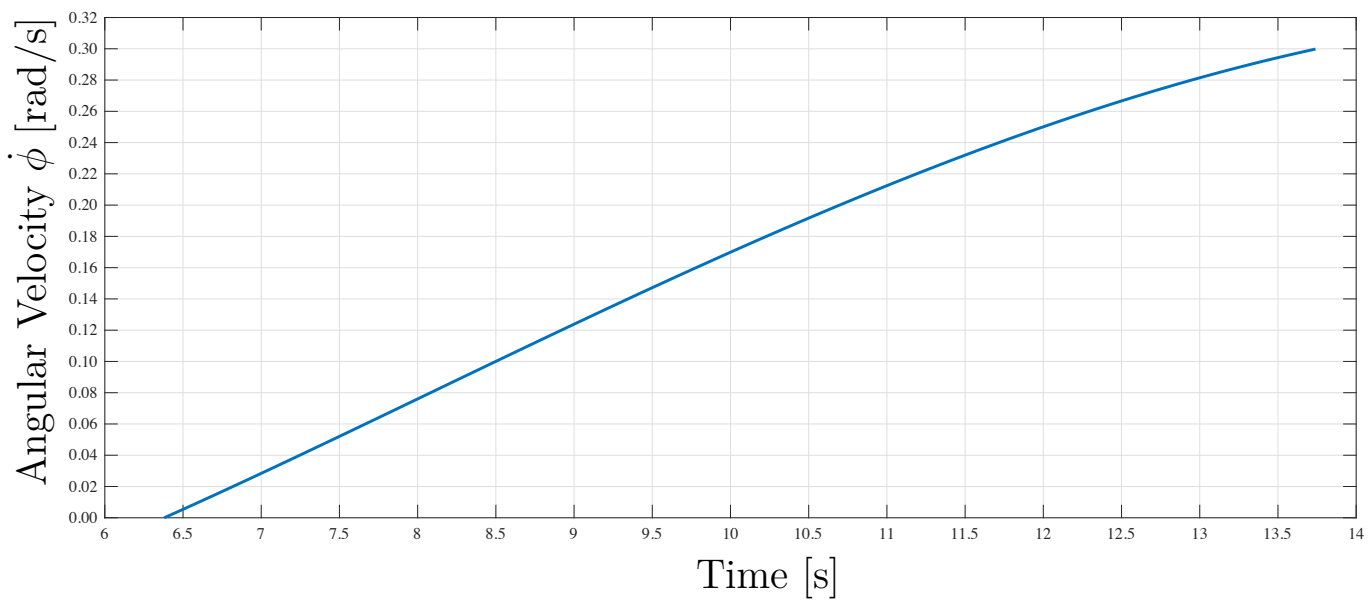

Figure 20: The angular velocity with respect to time from $6.4 \mathrm{~s}$ coasting to $13.6 \mathrm{~s}$.

As the vehicle's spin rate is increased the AoA of the fins is also increased. The tangential velocity is found through the radius multiplied by the angular velocity. At the fin tip this velocity is expected to be approximately $0.08 \mathrm{~m} / \mathrm{s}$. The resulting AoA at this angular velocity and $10 \mathrm{~m} / \mathrm{s}$ forward velocity is estimated to reach a maximum of $0.46^{\circ}$ at the fin tips. 


\section{Chapter 5}

\section{Conclusions and Recommendations}

\subsection{Conclusions}

Ultimately, an a-physical boundary condition implemented into a CFD simulation as a $5 \mathrm{~mm}$ section of a flat plate with a $-5 \mathrm{~m} / \mathrm{s}$ surface velocity is capable of representing a DBD plasma actuator's induced velocity profiles. The induced velocity profile measured $7 \mathrm{~mm}$ from the boundary condition fits closely to that of empirical data collected by Akansu [21]. Akansu's results are seen to be representative of an induced velocity profile of a DBD plasma actuator. Similar profiles are shown in various other works [35][36][38].

Simulations of an SDBD plasma actuator on a rocket fin at 14 separate locations at free-stream velocities of $20 \mathrm{~m} / \mathrm{s}$ and $70 \mathrm{~m} / \mathrm{s}$ yields a location of greatest force between $40 \%$ and $45 \%$ of the fin chord. These experiments show that as the velocity increases the local maximum stretches to include a wider range of chordwise locations. This increase in the local maximum begins to include the $50 \%$ chordwise 
location that is representative of the as built fin.

Using the $50 \%$ chord location, to represent the as built fin, through free-stream velocities of $10 \mathrm{~m} / \mathrm{s}$ to $90 \mathrm{~m} / \mathrm{s}$ in $10 \mathrm{~m} / \mathrm{s}$ increments, a maximum force of 52.5 $\mathrm{mN} / \mathrm{m}$ for a free stream velocity of $70 \mathrm{~m} / \mathrm{s}$ is predicted. The line of best fit through the examined velocities is shown to follow a second order polynomial curve. The force per span-wise distance with respect to velocity is shown to have lower applied forces at lower velocity increasing to a potential limit at higher velocities.

The force per span-wise distance with respect to velocity curve can be made to provide the angular acceleration with respect to velocity curve. This new second order polynomial equation is paired with the estimated velocity profile of the UNB sounding rocket. This produces the angular acceleration with respect to time curve. Through simple integration of the angular acceleration the angular velocity or roll rate of the sounding rocket is found. Through the range of examined velocities it is shown that the DBD plasma actuators are capable of inducing a roll rate on the sounding rocket of approximately $0.30 \mathrm{rad} / \mathrm{s}(2.86 \mathrm{RPM})$. Although small, this roll rate is observable by the IMUs on board the sounding rocket and will therefore be measurable. 


\subsection{Recommendations}

It is recommended that the tune-ability of the simplified boundary condition be examined in further detail. Variations of the boundary condition length and surface velocity can be examined to find how these parameters effect the induced velocity profile. This investigation can examine how these parameters effect the velocity profiles and the comparison to different configurations of empirical DBD plasma actuators.

It is recommended that a study similar to the one examined here examine free stream velocities ranging across the entirety of the rocket trajectory, if evidence of the functionality of DBD plasma actuators at these high velocities can be found. This experimentation will provide a better understanding of the angular velocity of the sounding rocket throughout the entirety of its flight. For this to be accomplished a solver that incorporates the compressibility of air is required. Solvers within the OpenFOAM package such as rhoSimpleFoam or rhoCentralFoam would be capable of examining this velocity range.

Three dimensional simulations with the boundary condition implemented on the rocket fin are recommended to examine if the introduction of span-wise flow across the surface of the fin alters the forces applied to the fin. This may be difficult as the necessity to keep the $\mathrm{y}^{+}$value less than one will require a fine mesh with a high number of cells. Thus, a computer with sufficient computational power is required. 
Wind tunnel tests of the fin and actuator should be performed with direct force measurements to compare the predicted forces found in the simulations to experiment. A wind tunnel analysis can provide a more controlled environment than a sounding rocket field test collecting potentially more extensive and more accurate results with a higher degree of repeatability. 


\section{Bibliography}

[1] B. Dunbar and L. Jenner. "Sounding rockets overview." (2017).

[2] A. Labergue, L. Leger, E. Moreau, and G. Touchard. "Effect of a plasma actuator on an airflow along an inclined wall: P.i.v. and wall pressure measurements." Journal of Electrostatics 63, 961-967 (2005).

[3] C. L. Enloe, T. E. McLaughlin, R. D. VanDyken, and J. C. Fischer. "Plasma structure in the aerodynamic plasma actuator." In "42nd AIAA Aerospace Sciences Meeting and Exhibit," American Institute of Aeronautics and Astronautics (2004).

[4] C. Enloe, T. E. McLaughlin, R. D. VanDyken, K. Kachner, E. J. Jumper, and T. C. Corke. "Mechanisms and responses of a single dielectric barrier plasma actuator: Plasma morphology." American Institute of Aeronautics and Astronautics 42(3) (2004).

[5] C. Enloe, T. E. McLaughlin, R. D. VanDyken, K. Kachner, E. J. Jumper, T. C. Corke, M. Post, and O. Haddad. "Mechanisms and responses of a single dielectric barrier plasma actuator: Geometric effects." American Institute of Aeronautics and Astronautics 42(3) (2004). 
[6] T. C. Corke, M. L. Post, and D. M. Orlov. "Single-dielectric barrier discharge plasma enhanced aerodynamics: Concepts optimization and applications." Journal of Propulsion and Power 24(8) (2008).

[7] M. Post. Plasma Actuators for Separation Control on Stationary and Unstationary Airfoils. Ph.D. thesis, University of Notre Dame (2004).

[8] T. Corke, C. Enloe, and S. P. Wilkinson. "Dielectric barrier discharge plasma actuators for flow control." Annual Review of Fluid Mechanics 42, 505-529 (2009).

[9] C. He, T. C. Corke, and M. P. Patel. "Plasma flaps and slats: An application of weakly ionized plasma actuators." Journal of Aircraft 46(3), 864-873 (2009).

[10] T. N. Jukes and K.-S. Choi. "Dielectric-barrier-discharge vortex generators: Characterization and optimization for flow separation control." Experimental Fluids 52, 329-345 (2011).

[11] T. N. Jukes and K.-S. Choi. "On the formation of streamwise vortices by plasma vortex generators." Journal of Fluid Mechanics 733, 370-393 (2013).

[12] P. Zhang, A. Liu, and J. Wang. "Flow structures in flat plate boundary layer induced by pulsed plasma actuator." Science China Technological Science 53(10), $2772-2782(2010)$.

[13] J. R. Roth, D. M. Sherman, and S. P. Wilkinson. "Electrohydrodynamic flow control with a glow-discharge surface plasma." American Institute of Aeronautics and Astronautics 38(7), 1166-1172 (2000). 
[14] Y. Jianyang, L. Huaping, and C. F. ZU Dimeng. "Investigation of the dbd plasma effect on flat plate flow." Plasma Science and Technology 16(3), 197$202(2014)$.

[15] Y. Jianyang, C. Fu, L. Huaping, and S. Yanping. "Investigation of the flow structure on a flat plate induced by unsteady plasma actuation with dns methods." Plasma Science and Technology 17(12), 1032-1037 (2015).

[16] J. R. Roth, D. M. Sherman, and S. P. Wilkinson. "Boundary layer flow control with a one atmosphere uniform glow discharge surface plasma." In "36th American Institute of Aeronautics and Astronautics meeting in Reno Nevada," 98-0328. American Institute of Aeronautics and Astronautics (1998).

[17] E. Moreau, L. Leger, and G. Touchard. "Effect of a dc surface-corona discharge on a flat plate boundary layer for air flow velocity up to $25 \mathrm{~m} / \mathrm{s} . "$ Journal of Electrostatics 64, 215-225 (2006).

[18] N. Bernard, J. Jolibois, and E. Moreau. "Lift and drag performances on axisymmetric airfoil controlled by plasma actuator." Journal of Electrostatics 67, $133-139$ (2009).

[19] D. Greenblatt, B. Goksel, I. Rechenberge, C. Schule, D. Romann, and C. Paschereit. "Dielectric barrier discharge flow control at very low flight reynolds numbers." American Institute of Aeronautics and Astronautics 46(6) (2008).

[20] H. R. Velkoff and J. Ketcham. "Effect of an electrostatic field on boundary-layer 
transition." American Institute of Aeronautics and Astronautics 6(7), 1381-1383 (1968).

[21] Y. Akansu, F. Karakaya, and A. Sanhsoy. "Active control of flow around naca 0015 airfoil by using dbd plasma actuator." In "EPJ Web of Conferences," volume 45 (2013).

[22] M. L. Post and T. C. Corke. "Separation control on high angle of attack airfoil using plasma actuators." American Institute of Aeronautics and Astronautics 42(11) (2004).

[23] M. L. Post and T. C. Corke. "Separation control using plasma actuators: Dynamic stall vortex control on oscillating airfoil." American Institute of Aeronautics and Astronautics 44(12) (2006).

[24] R. Sosa and G. Artana. "Steady control of laminar separation over airfoils with plasma sheet actuators." Journal of Electrostatics 64, 604-610 (2006).

[25] D. M. Schatzman and F. O. Thomas. "Turbulent boundary-layer separation control with single dielectric barrier discharge plasma actuators." American Institute of Aeronautics and Astronautics 48(8) (2010).

[26] J. Lopera, T. T. Ng, M. P. Patel, S. Vasundevan, and E. Santavicca. "Aerodynamic control using windward-surface plasma actuators on a separation ramp." Journal of Aircraft 44(6) (2007).

[27] A. Maslov, A. A. Sidorenko, B. Y. Zanin, B. V. Postnikov, and A. D. Budovsky. "Plasma control of flow separation on swept wing at high angles of attack." In "46th AIAA Aerospace Sciences Meeting and Exhibit," (2008). 
[28] D. Greenblatt, Y. Kastantin, C. Nayeri, and C. Paschereit. "Delta-wing flow control using dielectric barrier discharge actuators." American Institute of Aeronautics and Astronautics Journal 46(6) (2008).

[29] A. A. Sidorenko, A. D. Budovsky, A. Maslov, B. V. Postnikov, B. Y. Zanin, I. D. Zverkov, and V. V. Kozlov. "Plasma control of vortex flow on a delta wing at high angles of attack." Experimental Fluids 54(8) (2013).

[30] N. Bernard, P. Braud, G. Touchard, and E. Moreau. "Detachment and attachment of axisymmetric non-reactive jet with turbulent shear layer: Control by plasma actuator." Experimental Thermal and Fluid Science 32, 1193-1203 (2008).

[31] A. Labergue, L. Leger, E. Moreau, G. Touchard, and J.-P. Bonnet. "Experimental study of the detachment and the reattachment of an airflow along an inclined wall controlled by a surface corona discharge: Application to a plane turbulent mixing layer." IEEE Transactions on Industry Applications 40(5) (2004).

[32] A. Labergue, E. Moreau, N. Zouzou, and G. Touchard. "Separation control using plasma actuators: Application to a free turbulent jet." Journal of Physics D: Applied Physics 40(3) (2007).

[33] A. A. Sidorenko, A. D. Budovsky, A. V. Pushkarev, and A. Maslov. "Flight testing of dbd plasma separation control system." In "46th AIAA Aerospace Sciences Meeting and Exhibit," American Institute of Aeronautics and Astronautics (2008). 
[34] R. VanDyken, T. E. McLaughlin, and C. L. Enloe. "Parametric investigations of a single dielectric barrier plasma actuator." In "42nd AIAA Aerospace Sciences Meeting and Exhibit," American Institute of Aeronautics and Astronautics (2004).

[35] M. Forte, J. Jolibois, J. Pons, E. Moreau, G. Touchard, and M. Cazalens. "Optimization of a dielectric barrier discharge actuator by stationary and nonstationary measurements of the induced flow velocity: Application to airflow control." Experimental Fluids 43, 917-928 (2007).

[36] R. J. Roth and X. Dai. "Optimization of the aerodynamic plasma actuator as an electrohydrodynamic (ehd) electrical device." In "44th AIAA Aerospace Sciences Meeting and Exhibit," American Institute of Aeronautics and Astronautics (2006).

[37] J. Pons, E. Moreau, and G. Touchard. "Asymmetric surface dielectric barrier discharge in air at atmospheric pressure: Electrical properties and induced airflow characteristics." Journal of Physics D: Applied Physics 38, 3635-3642 (2005).

[38] J. Jolibois, M. Forte, and E. Moreau. "Application of an ac barrier discharge actuator to control airflow separation above a naca 0015 airfoil: Optimization of the actuation location along the chord." Journal of Electrostatics 66, 496-503 (2008).

[39] M. P. Patel, T. T. Ng, S. Vasudevan, and C. He. "Plasma actuators for hingeless 
aerodynamic control of an unmanned air vehicle." Journal of Aircraft 44(4) (2007).

[40] D. Opaits, D. Roupassov, S. Starikovskaia, A. Y. Starikovskii, and I. Zavialov. "Plasma control of boundary layer using low-temperature non-equilibrium plasma of gas discharge." In "43rd AIAA Aerospace Sciences Meeting and Exhibit," pages 2005-1180. American Institute of Aeronautics and Astronautics (2005).

[41] M. P. Patel, T. T. Ng, S. Vasudevan, M. L. Post, T. E. McLaughlin, and C. F. Suchomel. "Scaling effects of an aerodynamic plasma actuator." Journal of Aircraft 45(1) (2008).

[42] R. C. Nelson, T. C. Corke, C. He, H. Othman, T. Matsuno, M. P. Patel, and T. T. Ng. "Modification of the flow structure over a uav wing for roll control." In "45th AIAA Sciences Meeting and Exhibit," American Institute of Aeronautics and Astronautics (2007).

[43] A. N. Vorobiev, R. M. Rennie, E. J. Jumper, and T. E. McLaughlin. "Experimental investigations of lift enhancement and roll control using plasma actuators." Journal of Aircraft 45(4) (2008).

[44] C. Greenshields. "Openfoam version 2.4.x user guide." https://cfd.direct/openfoam/user-guide/ (2016).

[45] J. D. Anderson. Computational Fluid Dynamics: The Basics with Applications. McGraw Hill (1995). 
[46] R. J. Leveque. Finite-Volume Methods for Hyperbolic Problems. Cambridge University Press (2002).

[47] H. Versteeg and W. Malalasekera. An Introduction to Computational Fluid Dynamics The Finite Volume Method. Pearson Education Limited, Edinburgh Gate, Harlow, Essex England, 2 edition (2007).

[48] F. Menter. "Two-equation eddy-viscosity turbulence models for engineering applications." AIAA Journal 32(8) (1994).

[49] E. Moreau. "Airflow control by non-thermal plasma actuators." Journal of Physics D: Applied Physics 40, 605-636 (2007).

[50] W. Shyy, B. Jayaraman, and A. Anderson. "Modeling of glow discharge-induced fluid dynamics." Journal of Applied Physics 92(11) (2002).

[51] J. A. Schetz. Boundary Layer Analysis. Prentice Hall, Upper Saddle River, New Jersey (1993).

[52] F. M. White. Fluid Mechanics, volume 7th Edition. McGraw Hill, 1221 Avenue of the Americas, New York, NY 10020. (2011).

[53] S. F. Hoerner. Fluid-dynamic Drag: Practical Information on Aerodynamic Drag and Hydrodynamic Resistance, 2nd edition (1958).

[54] R. Monaghan. "A review and assessment of various formulae for turbulent skin friction in compressible flow." Technical Report 142, Aeronautical Research Council (1953). 